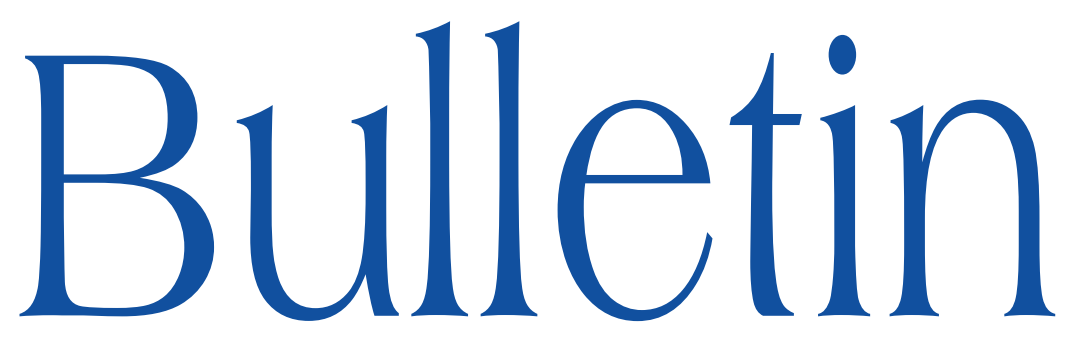

de la SOCIÉTÉ MATHÉMATIQUE DE FRANCE

\title{
SOUS-GROUPES $H$-LOXODROMIQUES
}

\author{
Antonin Guilloux
}

\section{Tome 139 \\ Fascicule 2}

2011

\section{SOCIÉTÉ MATHÉMATIQUE DE FRANCE}

Publié avec le concours du Centre national de la recherche scientifique 


\title{
SOUS-GROUPES $H$-LOXODROMIQUES
}

\author{
par Antonin Guilloux
}

\begin{abstract}
RÉsumé. - On considère une extension finie $k$ de $\mathbb{Q}_{p}$, avec $p$ un nombre premier, $H$ un sous-groupe d'indice fini de $k^{*}$ et le groupe $\mathrm{SL}(n, k)$.

Nous montrons que $\mathrm{SL}(n, k)$ admet un sous-groupe $\mathbb{Q}_{p}$-Zariski-dense dont toutes les matrices ont leur spectre inclus dans $H$ si et seulement si soit -1 est dans le sous-groupe $H$, soit $n$ n'est pas congru à 2 modulo 4 .

Abstract ( $H$-loxodromic subgroups). - Consider $k$ a finite extension of $\mathbb{Q}_{p}$, with $p$ a prime number. Let $H$ be a finite index subgroup of $k^{*}$ and $G$ be the group $\operatorname{SL}(n, k)$ with its Zariski topology of $\mathbb{Q}_{p}$-group. We investigate the existence of a subgroup of $G$ which is Zariski-dense and such that each of its elements has a spectrum included in $H$.

A necessary and sufficient condition is obtained: such a subgroup exists if and only if either -1 belongs to $H$ or the dimension $\mathrm{n}$ is not congruent to 2 modulo 4 .
\end{abstract}

\section{Introduction}

Dans ce texte, nous les sous-groupes de $\operatorname{SL}(n, k)$ - où $k$ est une extension finie de $\mathbb{Q}_{p}$ — dont toutes les matrices ont leur spectre inclus dans un sousgroupe d'indice fini $H$ de $k^{*}$. Nous arrivons au résultat d'existence suivant :

Texte reçu le 23 février 2007, révisé les 16 décembre 2008, 6 novembre 2009 et 2 décembre 2009, accepté le 5 octobre 2010

Antonin Guilloux, Unité de Mathématiques Pures et Appliquées, ENS Lyon, 46 allée d'Italie, 69007 Lyon, France • E-mail : antonin.guilloux@umpa.ens-lyon.fr Classification mathématique par sujets (2000). — 14L35, 20 G30.

Mots clefs. - Proximalité, loxodromie, corps $p$-adiques. 
ThÉORÈme 1.1. - Soient $n$ un entier supérieur $\grave{a} 2, k$ une extension finie de $\mathbb{Q}_{p}$, où $p$ est un nombre premier, et $H$ un sous-groupe d'indice fini de $k^{*}$.

Alors $\mathrm{SL}(n, k)$ admet un sous-groupe $\mathbb{Q}_{p}$-Zariski-dense dont toutes les matrices ont toutes leurs valeurs propres dans $H$ si et seulement si ou bien -1 est dans $H$, ou bien $n$ 'est pas congru à 2 modulo 4 .

Remarque 1.2. - Dans tout ce texte, nous considérons $\operatorname{SL}(n, k)$ comme un $\mathbb{Q}_{p}$-groupe et il hérite de ce fait d'une topologie de Zariski. Ce n'est pas sa topologie de Zariski de $k$-groupe que nous ne considérerons jamais. Pour éviter toute confusion, nous utiliserons les expressions $« \mathbb{Q}_{p}$-Zariski-dense », « $\mathbb{Q}_{p}$-fermé de Zariski », etc.

En guise d'illustration de ce théorème, explicitons-le dans un cas simple :

Corollaire 1.3. - On peut trouver dans $\mathrm{SL}_{n}\left(\mathbb{Q}_{p}\right)$ un sous-groupe Zariskidense dont toutes les valeurs propres sont des carrés si et seulement si $n$ n'est pas congru à 2 modulo 4 ou bien p est congru à 1 modulo 4.

Dans l'article «Automorphismes des cônes convexes»[4], Yves Benoist démontre notamment que $\operatorname{SL}(n, \mathbb{R})$ admet un sous-groupe Zariski-dense dont toutes les matrices ont leur spectre inclus dans $\mathbb{R}^{+*}$ si et seulement si $n$ n'est pas congru à 2 modulo 4 . Notre résultat en est un analogue dans le cadre des corps locaux non-archimédiens. On remarque que le fait de se placer sur un corps local non-archimédien ne change pas la condition donnée dans [4] pour le cas réel, sauf si -1 est dans $H$, auquel cas il n'y a aucune obstruction à l'existence de ces sous-groupes.

Mentionnons aussi que dans le cas réel, les sous-groupes de $\operatorname{SL}(n, k)$ préservant un cône convexe propre sont l'objet de la belle théorie des convexes divisibles développée principalement par Y. Benoist $[5,6,7,8]$ et que ces sousgroupes ont toutes leurs valeurs propres positives. Il serait intéressant de disposer d'une notion géométrique analogue dans le cas $p$-adique; et le cas échéant de développer une théorie des cônes divisibles. Pour l'instant les outils géométriques pertinents m'échappent, et en premier lieu la notion de convexité. Précisons que l'étude faite dans cet article pour le groupe $\operatorname{SL}(n, k)$ pourrait être menée avec les mêmes outils pour d'autres groupes. J'ai préféré me restreindre, en l'absence de motivations géométriques bien établies, à ce cas particulier où les idées à mettre en œuvre apparaissent bien.

L'ingrédient principal de la preuve de ce théorème est l'étude des éléments proximaux (i.e. ayant une valeur propre de module strictement dominant) et loxodromiques (toutes les valeurs propres de modules distincts) dans les groupes $p$-adiques. En effet, nous construisons, quand les conditions du théorème sont vérifiées, un sous-groupe $\mathbb{Q}_{p}$-Zariski-dense dont tous les éléments sont diagonalisables de valeurs propres de modules distincts.

TOME $139-2011-\mathrm{N}^{\circ} 2$ 
Les éléments proximaux dans le groupe spécial linéaire ont été beaucoup étudiés et utilisés. Citons en particulier les travaux de Gol'dsheid-Margulis [11], Abels-Margulis-Soifer [1] qui démontrent l'existence et l'abondance d'élément proximaux dans les sous-groupes Zariski-denses de $\mathrm{SL}(n, \mathbb{R})$ (et bien sûr d'autres groupes). Pour les éléments loxodromiques, on trouve ces résultats dans Abels-Margulis-Soifer [1], Benoist-Labourie [9] et Prasad [14].

Notre premier résultat est un analogue de ces travaux, à savoir qu'il existe des éléments loxodromiques en abondance dans les sous-groupes $\mathbb{Q}_{p}$-Zariskidenses de $\mathrm{SL}(n, k)$ dont les matrices sont trigonalisables sur $k$ :

ThÉORÈme 1.4. - Soit $k$ une extension finie de $\mathbb{Q}_{p}$. Si $\Gamma$ est un sous-groupe $\mathbb{Q}_{p}$-Zariski-dense de $\mathrm{SL}(n, k)$ dont toutes les matrices ont leur spectre inclus dans $k$, alors $\Gamma$ contient au moins un élément diagonalisable sur $k$ à valeurs propres de modules distincts.

De plus ces éléments forment un ensemble $\mathbb{Q}_{p}$-Zariski-dense de $\Gamma$.

Mentionnons que Platonov [13] obtient aussi des généralisations du théorème de Gol'dsheid-Margulis au cas $p$-adique, pour $\mathrm{SL}(n, k)$ et pour d'autres groupes algébriques. Cependant, notre résultat n'en découle pas directement. L'existence d'un élément loxodromique est prouvée dans la partie 2 (voir le théorème 2.5), et dans la partie 5 nous expliquons comment en déduire leur abondance (voir la proposition 5.7).

Dans la partie 3, nous définissons deux notions géométriques qui nous permettront de construire un groupe en contrôlant les valeurs propres des éléments. D'abord nous définissons et étudions la notion de $H$-valuation, qui est l'analogue de la notion de positivité dans [4]. Nous étudions notamment comment le fait que toutes les valeurs propres des matrices d'un groupe soient dans $H$ peut se comprendre avec cette notion. L'autre outil, que nous appelons $H$-sphère, est un revêtement fini bien choisi de l'espace projectif. Ces deux outils permettent d'appliquer des arguments de type ping-pong, à la Tits [15], pour construire des groupes de matrices diagonalisables en contrôlant leurs valeurs propres.

Cela nous mène à essayer de contrôler tout d'abord la valeur propre de plus grand module. Nous obtenons le théorème suivant :

THÉORÈme 1.5. - On peut trouver dans $\mathrm{SL}(n, k)$ un sous-groupe $\mathbb{Q}_{p}$-Zariskidense avec au moins un élément proximal et dont tout élément proximal a sa valeur propre de plus grand module dans $H$ si et seulement si on a ou bien -1 est dans $H$ ou bien $n$ est strictement plus grand que 2 .

De plus, dans ce cas $\mathrm{SL}(n, k)$ contient un sous-groupe $\mathbb{Q}_{p}$-Zariski-dense dont tous les éléments sont proximaux avec leur valeur propre de plus grand module dans $H$. 
Ce théorème est l'objet de la partie 4 ; plus précisément, il découle du théorème 4.2 .

Enfin, dans la partie 5, nous nous attelons au cour du problème, à savoir le contrôle de toutes les valeurs propres simultanément. Nous obtenons le théorème 5.1 , qui implique le théorème suivant :

THÉORÈmE 1.6. - On peut trouver dans $\mathrm{SL}(n, k)$ un sous-groupe $\mathbb{Q}_{p}$-Zariskidense avec au moins un élément loxodromique et dont tous les éléments loxodromiques sont à valeurs propres dans $H$ si et seulement si on a ou bien -1 est dans $H$ ou bien $n$ n'est pas congru à 2 modulo 4 .

De plus, dans ce cas $\mathrm{SL}(n, k)$ contient un sous-groupe $\mathbb{Q}_{p}$-Zariski-dense dont tous les éléments sont diagonalisables avec leurs valeur propres dans $H$ de modules distincts.

Les preuves de ces deux derniers théorèmes sont similaires. La nécessité de la condition n'est pas difficile à établir. Et dans le cas où cette condition est remplie, il faut soigneusement choisir des éléments de $\operatorname{SL}(n, k)$ avec lesquels jouer au ping-pong en étant sûr que toutes les valeurs propres de tous les produits seront dans $H$.

Le théorème 1.1 est une conséquence directe des théorèmes 1.4 et 1.6 : on cherche un sous-groupe $\mathbb{Q}_{p}$-Zariski-dense de $\mathrm{SL}(n, k)$ dont tous les éléments ont toutes leurs valeurs propres dans $H$. Tous ces éléments sont en particulier trigonalisables. Donc, le théorème 1.4 nous assure qu'un tel sous-groupe, s'il existe, contient un élément diagonalisable, à valeurs propres de modules distincts et dans $H$. Le théorème 1.6 nous indique alors qu'il faut et qu'il suffit que -1 soit dans $H$ ou bien que $n$ ne soit pas congru à 2 modulo 4 .

Cadre de la preuve. - Nous définissons ici les objets qui nous serviront tout au long du texte.

Dans toute la suite, $k$ désignera $\mathbb{Q}_{p}$ ou une extension finie de $\mathbb{Q}_{p}$ - donc un corps local muni d'une valeur absolue notée $||-., H$ un sous-groupe d'indice fini de $k^{*}$, et $V$ sera un $k$-espace vectoriel de dimension $n$. On notera $\mathbb{P}(V)$ l'espace projectif associé à $V$ et $V^{*}$ son dual. On notera $d$ l'indice de $H$ dans $k^{*}$, et $S=k^{*} / H$. On remarque que $H$ est à la fois ouvert et fermé dans $k^{*}{ }^{(1)}$.

On définit aussi l'espace $\mathbb{Q}(V)$ des hyperplans pointés : tout élément $a$ de $\mathbb{P}(V) \times \mathbb{P}\left(V^{*}\right)$ est vu comme un couple $\left(k v_{a}, V_{a}\right)$ où $v_{a}$ est un élément de $V$, et $V_{a}$ est un hyperplan de $V$. On note alors $\mathbb{Q}(V)=\left\{a=\left(k v_{a}, V_{a}\right) \in \mathbb{P}(V) \times\right.$ $\left.\mathbb{P}\left(V^{*}\right) / v_{a} \in V_{a}\right\}$. Enfin on note $X$ la variété des drapeaux de $V$.

(1) En effet il est d'intérieur non vide car une union finie de translatés de $H$ couvre $k^{*}$. C'est un groupe, il est donc ouvert. Son complémentaire est une union finie de translatés de $H$, donc est lui aussi ouvert, et $H$ est fermé.

TOME $139-2011-\mathrm{N}^{\mathrm{O}} 2$ 
On travaillera avec des actions de $\mathrm{GL}(V)$ sur les espaces $\mathbb{P}(V), \mathbb{P}\left(V^{*}\right), \mathbb{Q}(V)$ et $X$. Ce sont les actions naturelles induites par l'action de $\mathrm{GL}(V)$ sur $V$.

Insistons ici encore sur le fait que nous voyons le groupe $\operatorname{SL}(n, k)$ comme un $\mathbb{Q}_{p}$-groupe, équipé de sa topologie de Zariski ( $k$ est une extension de $\mathbb{Q}_{p}$ ).

\section{Proximalité}

2.1. Définitions. - Nous rappelons ici des définitions et résultats sur les actions proximales. On se restreint aux sous-groupes de $\mathrm{GL}(n, k)$, ce qui amène quelques simplifications dans les énoncés. Ces actions sont étudiées et utilisées par de nombreux auteurs, notamment par Tits [15], Abels, Margulis et Soifer [1], et Benoist [2, 3].

Soit $g$ dans $\mathrm{GL}(V)$. On note $\lambda_{1}(g), \ldots, \lambda_{n}(g)$ les valeurs propres de $g$ dans la clôture algébrique de $k$ répétées avec multiplicité avec $\left|\lambda_{1}\right| \geq\left|\lambda_{2}\right| \cdots \geq\left|\lambda_{n}\right|$. On peut alors définir les notions de proximalité et biproximalité :

\section{DÉfinition 2.1. - Proximalité}

- On dit qu'un élément $g$ de $\mathrm{GL}(V)$ est proximal si $\lambda_{1}(g)$ appartient à $k$ et $\left|\lambda_{1}(g)\right|>\left|\lambda_{2}(g)\right|$

- on dit qu'un sous-groupe $\Gamma$ de GL $(V)$ est proximal s'il contient un élément proximal.

\section{DÉFINITION 2.2. - Biproximalité}

On dit que $g$ est bi-proximal si $g$ et $g^{-1}$ sont proximaux

On s'intéresse ici non seulement à la première valeur propre, mais aussi à toutes les autres. Donc nous avons besoin de définir la notion d'éléments loxodromiques, de la manière suivante :

\section{DÉFINITION 2.3. - Loxodromie}

- On dit qu'un élément $g \in \mathrm{GL}(V)$ est loxodromique si tous les $\lambda_{i}(g)$ pour $1 \leq i \leq n$ sont dans $k$ et de valeurs absolues 2 à 2 distinctes,

- on dit qu'un sous-groupe $\Gamma$ de $\mathrm{GL}(V)$ est loxodromique s'il contient un élément loxodromique.

Remarque 2.4. - Pour un élément $g \in \mathrm{GL}(V), g$ est loxodromique si et seulement si son image $\Lambda^{m}(g)$ par la représentation de GL( $\left.V\right)$ dans $\Lambda^{m} V$ est proximale pour tout $m$ compris entre 1 et $n-1$.

Le théorème suivant réénonce la partie existence du théorème 1.4 : 
ThÉORÈme 2.5. - Soient $k$ une extension finie de $\mathbb{Q}_{p}$, et $\Gamma$ un sous-groupe Zariski-dense de $\mathrm{SL}(n, k)$. On suppose que tout élément $g$ de $\Gamma$ est trigonalisable sur $k$.

Alors $\Gamma$ est loxodromique.

On peut remarquer que nous avons besoin d'une hypothèse de trigonalisabilité, qui n'était pas nécessaire dans le cas réel. Nous ne pouvons pas nous en passer sur $\mathbb{Q}_{p}: \operatorname{SL}\left(n, \mathbb{Z}_{p}\right)$ est un sous-groupe Zariski-dense non loxodromique de $\operatorname{SL}\left(n, \mathbb{Q}_{p}\right)$.

La démonstration du théorème peut se décomposer en deux étapes. Dans un premier temps, nous supposons l'existence d'un sous-groupe $\Gamma$ qui vérifie les hypothèses du théorème, mais pas la conclusion. Nous arrivons alors à construire un sous-groupe $G$ d'un $\mathrm{SL}(d, k)$ qui est compact, $\mathbb{Q}_{p}$-Zariski-dense, et dont toutes les matrices sont trigonalisables sur $k$.

Dans un deuxième temps, nous montrons le lemme suivant :

Lemme 2.6. - Soient $d \geq 2$ un entier, et $G$ un sous-groupe compact, $\mathbb{Q}_{p}$-Zariski-dense de $\mathrm{SL}(d, k)$. Alors, $G$ possède des éléments non trigonalisables sur $k$.

Nous utiliserons dans cette preuve la notion de projecteur associé :

DÉFinition 2.7. - Si $g$ est un élément trigonalisable de $\operatorname{GL}(n, k)$, on appelle projecteur associé à $g$, noté $\pi_{g}$, le projecteur sur la somme des espaces caractéristiques de $g$ associés aux valeurs propres de module maximal parallèlement à la somme des autres espaces caractéristiques.

La propriété suivante est élémentaire et nous sera bien utile :

Propriété 1. - Soit $g$ un élément trigonalisable de $\operatorname{End}\left(k^{n}\right)$. Alors on peut trouver une suite $\left(c_{i}\right)_{i \in \mathbb{N}} d^{\prime}$ 'éléments de $k$ et une extraction de $c_{i} g^{i}$ qui converge vers un projecteur, qui est alors le projecteur $\pi_{g}$ associé $\grave{a} g$.

Démonstration. - $g$ est supposé trigonalisable : il admet donc une décomposition de Jordan $g=g_{s} g_{u}$ où $g_{s}$ est diagonalisable sur $k, g_{u}$ est nilpotent et ces deux éléments commutent.

On remarque dans un premier temps que la suite $\left(g_{u}^{p^{n}}\right)$ converge vers Id quand $n$ tend vers l'infini.

Ensuite, soit $c$ le maximum des valeurs absolues des valeurs propres de $g$. Alors, pour toute valeur propre $\lambda$ de $g$ de valeur absolue $c$, la grandeur $\frac{\lambda}{c}$ est de valeur absolue 1 . Donc le groupe engendré par $\left(\frac{\lambda}{c}\right)^{p^{n}}$ est compact, et on peut extraire une sous-suite $S$ tel que $\left(\frac{\lambda}{c}\right)^{p^{n}}$ tende vers 1 quand $n$ tend vers $\infty$ dans $S$.

TOME $139-2011-\mathrm{N}^{\mathrm{O}} 2$ 
Ainsi, quitte à extraire successivement, on construit une sous-suite $S$ telle qu'on ait la limite suivante :

$$
\frac{1}{c^{p^{n}}} g_{s}^{p^{n}} \underset{n \in S}{\stackrel{n \rightarrow \infty}{\longrightarrow}} \pi_{g}
$$

On en déduit la limite suivante :

$$
\frac{1}{c^{p^{n}}} g^{p^{n}} \underset{n \in S}{\stackrel{n \rightarrow \infty}{\longrightarrow}} \pi_{g}
$$

Supposons maintenant que l'on ait des constantes $c_{n}$, une extraction $S$, et un projecteur $\pi$ tels que $c_{n} g^{n} \stackrel{n \in S}{\longrightarrow} \pi$. Alors, on voit que le module de $c_{n}$ est équivalent à celui de $\lambda_{1}(g)^{n}$, par continuité du rayon spectral. Et $\pi$ est le projecteur associé à $g$.

L'énoncé est ainsi prouvé.

2.2. Construction d'un groupe compact. - Nous raisonnons par l'absurde, et cherchons à construire un sous-groupe compact d'un $\mathrm{SL}(d, k)$.

Soit $\Gamma$ un sous-groupe $\mathbb{Q}_{p}$-Zariski-dense de $\operatorname{SL}(n, k)$. On remarque tout d'abord que le groupe engendré par $\Gamma$ et les homothéties de $k^{n}$ est $\mathbb{Q}_{p}$-Zariskidense dans $\operatorname{GL}(n, k)$. Donc, dorénavant, $\Gamma$ désignera un sous-groupe $\mathbb{Q}_{p}$-Zariskidense de $\mathrm{GL}(n, k)$ qui contient les homothéties.

On suppose donc que tous les éléments de $\Gamma$ sont trigonalisables sur $k$, mais que $\Gamma$ n'est pas loxodromique. Le fait d'avoir rajouté les homothéties dans le groupe ne change pas ces propriétés.

De plus, on note $V$ l'espace vectoriel $k^{n}$.

2.2.1. Action sur $\Lambda^{\frac{n(n-1)}{2}}(\operatorname{End}(V))$. - Pour tout $g$ dans $\mathrm{GL}(n, k)$, on peut définir son action adjointe sur $\operatorname{End}(V)$ par $A d(g)(A)=g A g^{-1}$, puis $\rho(g)=$ $\Lambda^{\frac{n(n-1)}{2}}(A d(g))$ l'action induite de $\mathrm{g}$ sur $V^{\prime}=\Lambda^{\frac{n(n-1)}{2}}(\operatorname{End}(V))$. On remarque alors le résultat suivant :

Lemme 2.8. - Pour tout $g$ dans $\mathrm{GL}(n, k), g$ est loxodromique si et seulement si $\rho(g)$ est proximal.

Démonstration. - En effet si les valeurs propres de $g$ sont notées (répétées avec leur multiplicité) $\lambda_{1}, \ldots, \lambda_{n}$, avec $\left|\lambda_{1}\right| \geq\left|\lambda_{2}\right| \geq \cdots \geq\left|\lambda_{n}\right|$ alors les modules des valeurs propres de $\rho(g)$ sont les produits $\mu_{I}=\prod_{(i, j) \in I} \frac{\left|\lambda_{i}\right|}{\left|\lambda_{j}\right|}$, où I est un ensemble de $\frac{n(n-1)}{2}$ couples d'entiers inférieurs à $\mathrm{n}$. On voit que $\mu_{I}$ est maximal pour $I=\{(i, j) / i<j\}$. Enfin aucun autre ensemble n'atteint cette valeur si et seulement si la suite $\left|\lambda_{1}\right| \geq\left|\lambda_{2}\right| \geq \cdots \geq\left|\lambda_{n}\right|$ est strictement décroissante. 
On remarque aussi que, si $g$ est trigonalisable sur $k$, alors $\rho(g)$ l'est aussi.

On note alors, pour $\gamma \in \mathrm{GL}(n, k), W(\gamma) \subset V^{\prime}$ la somme des espaces caractéristiques de $\rho(\gamma)$ pour les valeurs propres de module maximal, et $\mu(\gamma)$ la dimension de $W(\gamma)$. Soit $\mu=\inf \{\mu(\gamma), \gamma \in \Gamma\}$. On a supposé que $\Gamma$ n'est pas loxodromique, c'est à dire que $\mu \geq 2$.

Posons $\Gamma_{\mu}=\{g \in \Gamma$ tels que $\mu(g)=\mu\}$. Cet ensemble est non vide.

2.2.2. Restriction de l'action de $\Gamma$ à un sous-espace. - Nous allons maintenant « restreindre » l'action de $\Gamma$ à un sous-espace de $\Lambda^{\frac{n(n-1)}{2}}(\operatorname{End}(V))$, en vue d'obtenir un sous-groupe de $\mathrm{SL}(\mu, k)$.

Soit $g$ dans $\Gamma_{\mu}$. Considérons $\pi=\pi_{\rho(g)}$ le projecteur sur $W=W(g)$ parallèlement à la somme des autres espaces caractéristiques.

On définit $\phi$ de la façon suivante : si $h$ est dans $\mathrm{GL}(n, k)$, alors on pose $\phi(h)=\pi \rho(h) \pi$.

Soit $K$ le semigroupe engendré par $\phi(\Gamma)$. On peut considérer que $K$ est inclus dans $\operatorname{End}(W)$. De plus la $\mathbb{Q}_{p}$-adhérence de Zariski de $K$ contient $\phi(\mathrm{GL}(n, k))$ et donc est $\operatorname{End}(W)$.

Soient enfin $G^{\prime}$ le semigroupe $K \cap \operatorname{SL}(W)$, et $G$ son adhérence topologique.

On veut montrer que $G$ est un groupe compact, $\mathbb{Q}_{p}$-Zariski-dense et que tous ses éléments sont trigonalisables. Commençons par le lemme suivant :

Lemme 2.9. - Tout les éléments de $G$ sont trigonalisables.

Démonstration. - Tout d'abord, d'après la propriété 1 , il existe des constantes $c_{n}$ telles que $\pi$ est une valeur d'adhérence de $c_{n} \rho(g)^{n}$.

On considère donc un élément de $K$, qu'on peut écrire comme le produit $\phi\left(\gamma_{1}\right) \cdots \phi\left(\gamma_{l}\right)$. Alors cet élément est par construction une valeur d'adhérence de la suite $c_{n}^{2 l} \rho\left(\left(g^{n} \gamma_{1} g^{n}\right) \cdots\left(g^{n} \gamma_{l} g^{n}\right)\right)$. Tous les éléments de cette suite sont trigonalisables par hypothèse, donc toute valeur d'adhérence aussi.

Ainsi tous les éléments de $K$ sont trigonalisables, et ceux de $G$ sont limites d'éléments de $K$. On conclut enfin que tous les éléments de $G$ sont trigonalisables.

Ensuite on vérifie que $G$ est $\mathbb{Q}_{p}$-Zariski-dense dans $\operatorname{SL}(W)$. En effet le soussemigroupe des matrices de $K$ de déterminant égal à une puissance $\mu$-ième est toujours $\mathbb{Q}_{p}$-Zariski-dense dans $\operatorname{GL}(W)$, car il est d'indice fini. Or ce soussemigroupe est égal à $k^{*} \times G^{\prime}$. Donc $G^{\prime}$ est $\mathbb{Q}_{p}$-Zariski-dense dans $\operatorname{SL}(W)$, et $G$ aussi.

Il reste à montrer que $G$ est un groupe compact. La fin de cette partie est consacrée à la preuve de ce point.

Lemme 2.10. - Si g appartient à $K$, toutes ses valeurs propres sont de même module. 
Démonstration. - On raisonne par l'absurde : soit $g \in K$ tels que ses valeurs propres ne sont pas toutes de même module. On pose $g=\phi\left(g_{1}\right) \cdots \phi\left(g_{r}\right)$, avec $g_{1}, \ldots, g_{r} \in \Gamma$. On sait alors que la dimension $\nu$ de la somme des espaces caractéristiques associé aux valeurs propres de module maximal de l'endomorphisme $\pi \rho\left(g_{1}\right) \pi \rho\left(g_{2}\right) \cdots \pi \rho\left(g_{r}\right) \pi$ vérifie $\nu<\mu$.

Donc, en utilisant la propriété 1 , il existe un entier $n \in \mathbb{N}$ tel que :

$$
\mu\left(g^{n} g_{1} g^{n} g_{2} \cdots g^{n} g_{r} g^{n}\right)=\nu<\mu .
$$

Mais ceci est absurde car $g^{n} g_{1} g^{n} \cdots g_{r} g^{n}$ appartient à $\Gamma$.

Corollaire 2.11. - Tous les éléments de G ont leurs valeurs propres de module 1 .

Or le semigroupe $G$ agit irréductiblement, car il est $\mathbb{Q}_{p}$-Zariski-dense. Le lemme suivant nous montre alors qu'il est compact :

Lemme 2.12. - Soit $k$ une extension finie de $\mathbb{Q}_{p}$, et $G$ un semigroupe de $\operatorname{End}\left(k^{\mu}\right)$ qui agit irréductiblement sur $k^{\mu}$, dont toutes les valeurs propres sont de valeur absolue inférieure à 1 . Alors $G$ est relativement compact.

Démonstration. - Soit $A$ l'algèbre associative engendrée par $G$. Montrons que la trace, notée $T r$, est non dégénérée sur $A$.

Pour cela on remarque que, comme $k^{\mu}$ est un $G$-module simple, c'est aussi un $A$-module simple. C'est de plus un $A$-module fidèle, car $A$ est une sousalgèbre de $\operatorname{End}\left(k^{\mu}\right)$. Donc l'algèbre $A$ est simple, et on sait alors, d'après le lemme de Schur et le théorème de Wedderburn (cf. [12], pp. 643 et 649) que $A=\operatorname{End}_{L}\left(k^{\mu}\right)$, où $L=\operatorname{End}_{A}\left(k^{\mu}\right)$ est une algèbre à division.

Donc pour tout élément $a$ non nul de $A$, on trouve un $b$ dans $A$ tel que $a b$ soit un projecteur de rang 1 dans le $L$-espace vectoriel $k^{\mu}$. Et alors $\operatorname{Tr}(a b)=$ $[L, k] \neq 0$. La trace est donc bien non-dégénérée sur $A$.

Soient alors $\left(h_{i}\right)$ une base de $A$ formée d'éléments de $G,\left(e_{i}\right)$ sa base duale. Soit $g \in K$. Soit $\phi_{g}(x)=\operatorname{Tr}(g x)$.

On a bien $\phi_{g}=\sum \operatorname{Tr}\left(g h_{i}\right) e_{i}$. Or $g h_{i} \in G$ et $\left|\operatorname{Tr}\left(g h_{i}\right)\right| \leq \mu$ car toutes les valeurs propres sont inférieures à 1 en module. Donc la norme de la forme linéaire $\phi_{g}$ est bornée par $C \mu$ où $C$ est une constante. C'est-à-dire que la norme de $\phi_{g}$ est bornée indépendamment de $g$. Finalement, les coefficients de $g$ sont bornés en valeur absolue indépendamment de $g$.

On a bien montré que $G$ est relativement compact. 
Ici, $G$ étant de plus fermé, il est compact. Enfin $G$ est un groupe, car c'est un sous-semigroupe compact formé d'éléments inversibles ${ }^{(2)}$.

Pour résumer : on a construit un sous-groupe compact $\mathbb{Q}_{p}$-Zariski-dense de $\mathrm{SL}(W)$ dont toutes les matrices sont trigonalisables sur $k$.

La partie suivante montrera que cette situation est impossible.

2.3. Eléments non trigonalisables des sous-groupes compacts. - Le but de cette section est de montrer le lemme suivant, qui permet facilement de conclure le raisonnement par l'absurde :

Lemme 2.13. - Soit $G$ un sous-groupe compact $\mathbb{Q}_{p}$-Zariski-dense de $\operatorname{SL}(d, k)$, avec $d \geq 2$.

Alors $G$ contient une matrice non trigonalisable sur $k$.

Démonstration. - $G$ est un sous- $\mathbb{Q}_{p}$-groupe de Lie de $\mathrm{SL}(d, k)$, car c'est un sous-groupe compact. Soit donc $\mathscr{G}$ son algèbre de Lie ( sur $\left.\mathbb{Q}_{p}\right)$. C'est une sousalgèbre de Lie de $s l(d, k)$, la $\mathbb{Q}_{p}$-algèbre de Lie de $\mathrm{SL}(d, k)$. Elle est non réduite à 0 , sinon $G$ serait discret, donc fini, donc ne serait pas $\mathbb{Q}_{p}$-Zariski-dense.

De plus, comme $G$ est $\mathbb{Q}_{p}$-Zariski-dense dans $\mathrm{SL}(d, k), \mathscr{G}$ est un idéal de la $\mathbb{Q}_{p}$-algèbre $\operatorname{sl}(d, k)$. C'est l'objet du lemme suivant :

Lemme 2.14. - Soit $G \subset G^{\prime} \subset \mathrm{GL}(d, \mathbb{K})$ deux $\mathbb{K}$-groupes de Lie, où $\mathbb{K}$ est une extension finie de $\mathbb{Q}_{p}$. Soit $\mathscr{G}$ et $\mathscr{G}^{\prime}$ leur algèbre de Lie respective. Alors si $G$ est $\mathbb{Q}_{p}$-Zariski-dense dans $G^{\prime}, \mathscr{G}$ est un idéal de $\mathscr{G}^{\prime}$

Démonstration. - On sait que pour tout $h \in G, \operatorname{Ad}_{h}$ stabilise $\mathscr{G}$. Or cette propriété s'écrit comme l'annulation de polynômes en les coefficients de $h$.

On en déduit par $\mathbb{Q}_{p}$-Zariski-densité de $G$, que pour tout $g \in G^{\prime}, \operatorname{Ad}_{g}$ stabilise $\mathscr{G}$. Donc, pour tout $y \in \mathscr{G}^{\prime}$, on a $a d_{y}(x) \in \mathscr{G}$.

C'est à dire que $\mathscr{G}$ est un idéal de $\mathscr{G}^{\prime}$.

Or d'après [10, Paragraphe 6 , Numéro 10], la $\mathbb{Q}_{p}$-algèbre de Lie $s l(d, k)$ est simple. Donc $\mathscr{G}$ est tout $\operatorname{sl}(d, k)$.

Il suffit maintenant de remarquer que $s l(d, k)$ contient des matrices non trigonalisables sur $k$. Soit $x$ l'une d'entre elles. Quitte à multiplier $x$ par une puissance de $p$, on peut supposer que $x$ est dans le domaine de définition de l'exponentielle et que $\exp (x)$ est dans $G$.

Alors les matrices $x$ et $\exp (x)$ sont trigonalisables exactement sur le même corps : les valeurs propres de la première sont les logarithmes des valeurs propres de la seconde. On a choisi un élément $x$ non-trigonalisable sur $k$, donc $\exp (x)$ ne l'est pas non plus.

(2) En effet, si $g \in G$, alors il existe une sous-suite $\left(g^{n_{k}}\right)$ convergente de la suite $\left(g^{n}\right)_{n \in \mathbb{N}}$. Et la suite $\left(g^{n_{k+1}-n_{k}-1}\right)$ est une suite d'éléments de $G$ qui converge vers $g^{-1}$. 
Le lemme est ainsi démontré.

Ceci conclut la démonstration par l'absurde. On a bien démontré le théorème 2.5.

\section{Ensembles limites et $H$-valuation dans $\mathbb{Q}(V)$}

Nous disposons d'un outil pour étudier un groupe proximal $\Gamma$ : les ensembles limites de l'action du groupe sur les espaces $\mathbb{P}(V), \mathbb{Q}(V)$. Ils sont définis comme les plus petits fermés invariants par $\Gamma$ de ces espaces (voir la proposition 3.6 plus loin).

Nous introduirons ensuite la notion de $H$-valuation d'un ensemble de $\mathbb{Q}(V)$.

Cela permettra dans la prochaine partie de comprendre les conditions pour que tout élément $g$ proximal de $\Gamma$ vérifie $\lambda_{1}(g) \in H$.

3.1. Ensembles limites. - Nous donnons ici des résultats qui nous assurent l'existence de ces ensembles limites. Pour les énoncer, nous avons besoin de quelques définitions supplémentaires.

Soit $\Gamma$ un sous-groupe de $\mathrm{GL}(V)$. On dit qu'une partie $F$ de $V, \mathbb{P}(V), \mathbb{Q}(V) \ldots$ est $\Gamma$-invariante si pour tout $\gamma \in \Gamma$, on a $\gamma F \subset F$.

Si un élément $g$ de $\operatorname{GL}(n, k)$ est proximal, la droite propre de $V$ associée à $\lambda_{1}(g)$ et la droite propre de ${ }^{t} g$ associée à $\lambda_{1}(g)$ sont des points fixes attracteurs pour l'action de $g$ sur respectivement $\mathbb{P}(V)$ et $\mathbb{P}\left(V^{*}\right)$. Nous les utiliserons souvent, donc fixons des notations :

DÉfinition 3.1. - Si $g$ est un élément proximal de $\mathrm{GL}(n, k)$, on note $x_{g}^{+}$le point fixe attracteur de $g$ dans $\mathbb{P}(V)$ et $y_{g}^{-}$celui dans $\mathbb{P}\left(V^{*}\right)$.

De plus on note $Y_{g}^{-}$le noyau de $y_{g}^{-}$. C'est le supplémentaire $g$-invariant de $x_{g}^{+}$.

Le lemme suivant décrit la dynamique d'un élément $g$ proximal sur l'espace projectif :

Lemme 3.2. - Soit $g$ un élément proximal de $\mathrm{GL}(n, k)$, et notons $Y_{g}^{-} l$ l'hyperplan projectif correspondant au noyau de $y_{g}^{-}$. Alors l'action de $g$ sur $\mathbb{P}(V)$ admet $x_{g}^{+}$comme point fixe attracteur, de bassin d'attraction $\mathbb{P}(V) \backslash Y_{g}^{-}$.

Démonstration. - On vérifie que la suite $\left(\frac{1}{\lambda_{1}^{n}(g)} g^{n}\right)_{n \in \mathbb{N}}$ converge vers le projecteur sur la droite $x_{g}^{+}$parallèlement à $Y_{g}^{-}$, ce qui prouve le lemme.

Le lemme suivant fait un autre lien entre les éléments proximaux et les projecteurs de rang 1 : 
LEMme 3.3. - Soit $\pi$ un projecteur de rang 1 sur une droite $x \in \mathbb{P}(V)$ parallèlement à un hyperplan, que l'on voit comme le noyau d'une forme linéaire $y \in \mathbb{P}\left(V^{*}\right)$. Considérons $g_{n}$ une suite d'éléments de $\mathrm{GL}(n, k)$ qui converge vers $\pi$.

Alors pour $n$ suffisamment grand, $g_{n}$ est proximal, et de plus $x_{g_{n}}^{+}$tend vers $x$ et $y_{g_{n}}^{-}$tend vers $y$.

Démonstration. - Considérons l'action de $\pi \operatorname{sur} \mathbb{P}(V)$ : tout voisinage compact $\Omega$ de $x$ est envoyé sur le singleton $\{x\}$ qui est inclus dans $\Omega$. Donc pour $n$ suffisamment grand, $g_{n}$ envoie $\Omega$ dans $\Omega$. Donc l'action de $g_{n}$ admet un point fixe $x_{n}$ dans $\Omega$. C'est à dire que la droite $x_{n}$ est une droite propre pour $g_{n}$. De même pour tout voisinage $\Omega^{\prime}$ de $y$ dans $\mathbb{P}\left(V^{*}\right)$, pour $n$ suffisamment grand, $g_{n}$ admet un point fixe $y_{n}$ dans $\Omega^{\prime}$.

De plus par continuité, la valeur propre de $g_{n}$ associée à $x_{n}$ tend vers 1 , alors que le module des autres valeurs propres tend vers 0 .

Donc pour $n$ suffisamment grand, $g_{n}$ est proximal et $x_{n}=x_{g_{n}}^{+}, y_{n}=y_{g_{n}}^{-}$. On en déduit que $x_{g_{n}}^{+}$tend vers $x$ et $y_{g_{n}}^{-}$tend vers $y$.

Si $g$ est biproximal, on note $x_{g}^{-}=x_{g^{-1}}^{+}, y_{g}^{+}=y_{g^{-1}}^{-}$. Le point $\left(x_{g}^{+}, y_{g}^{+}\right)$est le point fixe attracteur de $g$ dans $\mathbb{Q}(V)$ :

DÉfinition 3.4. - Si $g$ est un élément biproximal de GL $(n, k)$, on note $a_{g}^{+}=$ $\left(x_{g}^{+}, y_{g}^{+}\right)$le point fixe attracteur de $g$ dans $\mathbb{Q}(V)$. De même, on note $a_{g}^{-}=$ $a_{g^{-1}}^{+}=\left(x_{g}^{-}, y_{g}^{-}\right)$le point fixe répulseur de $g$.

On définit la notion de partie transverse, sur laquelle on reviendra dans la partie suivante :

DÉfinition 3.5. - Une partie $\Lambda$ de $\mathbb{P}(V) \times \mathbb{P}\left(V^{*}\right)$ est dite transverse si pour deux éléments distincts $\left(k v_{1}, V_{1}\right)$ et $\left(k v_{2}, V_{2}\right)$ (où $v_{1}$ et $v_{2}$ sont deux vecteurs de $V$ et $V_{1}$ et $V_{2}$ deux hyperplans de $\left.V\right) v_{1}$ n'appartient pas à $V_{2}$.

On dispose de plus sur $V$ de la norme max dans une base fixée, que l'on notera $\|$.$\| . On peut alors en déduire de manière naturelle une norme sur V^{*}$ toujours notée $\|$.$\| , et des distances sur \mathbb{P}(V)$ et $\mathbb{Q}(V)$ que l'on note toutes les deux $d$. On définit la distance sur $\mathbb{P}(V)$ par la formule suivante :

$$
d(x, y)=\inf \{\|u-v\| \text { tel que } u \in x, v \in y,\|v\|=\|u\|=1\}
$$

On note que pour le choix de la norme fait, cet ensemble est non vide.

On dispose alors de la proposition suivante :

Proposition 3.6. - Soient $k$ une extension finie de $\mathbb{Q}_{p}$ et $\Gamma$ un sous-groupe $\mathbb{Q}_{p}$-Zariski-dense proximal de $\mathrm{SL}(n, k)$. Alors d'une part :

TOME $139-2011-\mathrm{N}^{\circ} 2$ 
1. L'ensemble $\Gamma_{\text {prox }}$ des éléments proximaux de $\Gamma$ est $\mathbb{Q}_{p}$-Zariski-dense dans $\Gamma$

2. Soit $\Lambda_{\Gamma}^{\mathbb{P}}$ l'adhérence de l'ensemble $\left\{x_{g}^{+} / g \in \Gamma_{\text {prox }}\right\}$. Tout fermé non vide $\Gamma$-invariant de $\mathbb{P}(V)$ contient $\Lambda_{\Gamma}^{\mathbb{P}}$. Donc l'action de $\Gamma$ sur $\Lambda_{\Gamma}^{\mathbb{P}}$ est minimale.

3. Soit $\Lambda_{\Gamma}^{\mathbb{P}^{*}}$ l'adhérence de l'ensemble $\left\{y_{g}^{-} / g \in \Gamma_{\text {prox }}\right\}$. Tout fermé non vide $\Gamma$-invariant de $\mathbb{P}\left(V^{*}\right)$ contient $\Lambda_{\Gamma}^{\mathbb{P}^{*}}$. Donc l'action de $\Gamma$ sur $\Lambda_{\Gamma}^{\mathbb{P}^{*}}$ est minimale.

4. Pour tous $\varepsilon>0, x^{+}$dans $\Lambda_{\Gamma}^{\mathbb{P}}$ et $y^{-}$dans $\Lambda_{\Gamma}^{\mathbb{P}^{*}}$, l'ensemble $\left\{g \in \Gamma_{\text {prox }} / d\left(x_{g}^{+}, x^{+}\right)<\varepsilon, d\left(y_{g}^{-}, y^{-}\right)<\varepsilon\right\}$ est encore Zariski-dense dans $\Gamma$.

5. $\Lambda_{\Gamma}^{\mathbb{P}} \times \Lambda_{\Gamma}^{\mathbb{P}^{*}}$ contient une partie transverse dense.

Et d'autre part :

(a) L'ensemble $\Gamma_{\text {bip }}$ des éléments biproximaux de $\Gamma$ est encore $\mathbb{Q}_{p}$-Zariskidense dans $\Gamma$

(b) Soit $\Lambda_{\Gamma}^{\mathbb{Q}}$ l'adhérence de l'ensemble $\left\{a_{g}^{+} / g \in \Gamma_{\text {bip }}\right\}$. Tout fermé non vide $\Gamma$-invariant de $\mathbb{Q}(V)$ contient $\Lambda_{\Gamma}^{\mathbb{Q}}$. Donc l'action de $\Gamma$ sur $\Lambda_{\Gamma}^{\mathbb{Q}}$ est minimale.

(c) Pour tous $\varepsilon>0, a^{+}$et $a^{-}$dans $\Lambda_{\Gamma}^{\mathbb{Q}}$, l'ensemble $\left\{g \in \Gamma_{\text {bip }} / d\left(a_{g}^{+}, a^{+}\right)<\varepsilon, d\left(a_{g}^{-}, a^{-}\right)<\varepsilon\right\}$ est encore $\mathbb{Q}_{p}$-Zariski-dense dans $\Gamma$.

(d) $\Lambda_{\Gamma}^{\mathbb{Q}}$ contient une partie dense et transverse.

Démonstration. - C'est une conséquence de la partie 3 de [3]. Plus précisément, la $\mathbb{Q}_{p}$-Zariski-densité de $\Gamma_{\text {prox }}$ et $\Gamma_{b i p}$ découle du corollaire 1 et les autres points du lemme 3.6.

Nous donnons ici la preuve des cinq premiers points pour donner une idée des arguments à mettre en jeu. La preuve des quatres derniers points est très similaire.

Commençons par prouver le point (2) :

Soit $F$ un fermé non vide $\Gamma$-invariant de $\mathbb{P}(V), x$ un point de $F$ et $g$ un élément de $\Gamma_{\text {prox }}$. Alors, par $\mathbb{Q}_{p}$-Zariski-densité de $\Gamma$, il existe $h \in \Gamma$ tel que $h(x)$ n'appartient pas au noyau de $y_{g}^{-}$, c'est à dire que $h(x)$ est dans le bassin d'attraction de $x_{g}^{+}$pour $g$. Donc $x_{g}^{+}=\lim _{n \rightarrow \infty} g^{n} h(x)$ appartient à $F$.

Ainsi $F$ contient tout $\Lambda_{\Gamma}^{\mathbb{P}}$.

Le point (3) se prouve de la même manière, en considérant l'action de $\Gamma$ sur $\mathbb{P}\left(V^{*}\right)$

Prouvons ensuite le point (4), qui implique le point (1) :

Soient $x^{+}$dans $\Lambda_{\Gamma}^{\mathbb{P}}$ et $y^{-}$dans $\Lambda_{\Gamma}^{\mathbb{P}^{*}}$ et $F$ un $\mathbb{Q}_{p}$-fermé de Zariski de $\Gamma$ contenant l'ensemble :

$$
\left\{g \in \Gamma_{\text {prox }} / d\left(x_{g}^{+}, x^{+}\right)<2 \varepsilon, d\left(y_{g}^{-}, y^{-}\right)<2 \varepsilon\right\} .
$$


Par définition, on trouve deux éléments $g$ et $h$ dans $\Gamma_{\text {prox }}$ tels qu'on a :

$$
d\left(x_{g}^{+}, x^{+}\right)<\varepsilon \quad \text { et } d\left(y_{h}^{-}, y^{-}\right)<\varepsilon .
$$

Soit $U$ le $\mathbb{Q}_{p}$-ouvert de Zariski des éléments $u$ de $\Gamma$ tels que $u\left(x_{h}^{+}\right)$n'est pas dans $\operatorname{Ker}\left(y_{g}^{-}\right)$. Comme $\Gamma$ est $\mathbb{Q}_{p}$-Zariski-dense, $U$ est non vide.

De plus, pour tout $u$ dans $U$, la suite $\left(\frac{1}{\lambda_{1}(g)^{n} \lambda_{1}(h)^{m}} g^{n} u h^{m}\right)_{n, m \in N}$ tend vers le projecteur de rang 1 sur la droite $x_{g}^{+}$parallèlement à l'hyperplan $\operatorname{Ker}\left(y_{h}^{-}\right)$ quand $n$ et $m$ tendent vers l'infini.

D'après le lemme 3.3, pour tout $u$ dans $U$, pour $n$ et $m$ suffisamment grand, l'élément $\gamma=g^{n} u h^{n}$ est proximal et de plus $d\left(x_{\gamma}^{+}, x^{+}\right)<2 \varepsilon$ et $d\left(y_{\gamma}^{-}, y^{-}\right)<2 \varepsilon$.

Donc $F$ contient tout $U^{(3)}$. Or $\Gamma$ est Zariski-dense dans $\operatorname{SL}(n, k)$ donc en particulier $\mathbb{Q}_{p}$-Zariski-connexe, ce qui permet de conclure que $F=\Gamma$.

Enfin pour le point (5) : on fixe une suite $\left(x_{n}, y_{n}\right)_{n \in \mathbb{N}}$ dense dans $\Lambda_{\Gamma}^{\mathbb{P}} \times \Lambda_{\Gamma}^{\mathbb{P}^{*}}$. Alors, grâce au point (4) que l'on vient de montrer, on peut construire par récurrence une suite $g_{n}$ d'éléments de $\Gamma_{\text {prox }}$ telle que :

- Pour tout $n$, on a $d\left(x_{g_{n}}^{+}, x_{n}\right)<\frac{1}{n}$, et $d\left(y_{g_{n}}^{-}, y_{n}\right) \leq \frac{1}{n}$.

- pour tout $n$, pour tout $j<n$, on a $y_{g_{j}}^{-}\left(x_{g_{n}}^{+}\right) \neq 0$ et $y_{g_{n}}^{-}\left(x_{g_{j}}^{+}\right) \neq 0$. Autrement dit, la partie $\left(x_{g_{j}}^{+}, y_{g_{j}}^{-}\right)_{j \leq n}$ est transverse.

On conclut en remarquant que la partie $\left(x_{g_{n}}^{+}, y_{g_{n}}^{-}\right)_{n \in \mathbb{N}}$ est dense et transverse dans $\Lambda_{\Gamma}^{\mathbb{P}} \times \Lambda_{\Gamma}^{\mathbb{P}^{*}}$.

Ces ensembles $\Lambda_{\Gamma}^{\mathbb{P}}$ et $\Lambda_{\Gamma}^{\mathbb{Q}}$ sont les ensembles limites de $\Gamma$ sur respectivement $\mathbb{P}(V)$ et $\mathbb{Q}(V)$. Le deuxième notamment nous sera très utile, une fois que nous aurons défini la notion de $H$-valuation d'une partie de $\mathbb{Q}(V)$.

3.2. $H$-valuation. - Nous introduisons dans cette partie la notion de $H$-valuation. Dans le cas de l'article d'Y. Benoist, cette notion — appelée positivité est reliée aux parties convexes de l'espace projectif. Mais ici nous ne disposons pas de convexité, et donc nous devons nous passer de cette vision des choses. Rappelons qu'on a noté $S$ le quotient $k^{*} / H$.

Soit $a$ un élément de $\mathbb{P}(V) \times \mathbb{P}\left(V^{*}\right)$. On note alors $a=\left(x_{a}, y_{a}\right)$, et on choisit arbitrairement des vecteurs non nuls $v_{a}$ et $f_{a}$ appartenant respectivement à $x_{a}$ et $y_{a}$. On définit $\eta_{a b}$ comme égal à 0 si $f_{a}\left(v_{b}\right)=0$, à la classe de $f_{a}\left(v_{b}\right)$ dans $S$ sinon; et $\zeta_{a b}=\eta_{a b}^{d-1} \eta_{b a}$ (on rappelle que $d$ est l'indice de $H$ dans $k^{*}$ ). Ces grandeurs dépendent des choix faits, mais nous n'utiliserons que des produits de ces grandeurs qui eux ne dépendront pas de ces choix.

(3) En effet considérons l'application $\phi$ de $\Gamma \times \Gamma$ dans $\Gamma$ qui à $a, b$ associe $a u b . \phi^{-1}(F)$ est un $\mathbb{Q}_{p}$-fermé de Zariski de $\Gamma$ et contient le semigroupe $\left(g^{n}, h^{m}\right)$ pour $n$ et $m$ assez grand. Comme l'adhérence de Zariski d'un semigroupe est un groupe, $\phi^{-1}(F)$ contient l'identité et $F$ contient $u$.

TOME $139-2011-\mathrm{N}^{\mathrm{O}} 2$ 
Soit $\Lambda$ une partie de $\mathbb{P}(V) \times \mathbb{P}\left(V^{*}\right)$. On voit alors que $\Lambda$ est transverse si pour tout couple $(a, b)$ d'éléments distincts de $\Lambda$, on a $f_{a}\left(v_{b}\right) \neq 0$. On définit la $H$-valuation d'une telle partie :

DÉfinition 3.7 ( $H$-valuation). - $\quad$ On dit que $\Lambda$ est $H$-valuée si on peut choisir pour tout $a \in \Lambda$ les vecteurs $v_{a}$ et $f_{a}$ de telle sorte que pour tout couple $(b, c)$ d'éléments de $\Lambda, f_{b}\left(v_{c}\right)$ appartient à $H \cup\{0\}$.

- Si $p$ est un entier plus grand que 2 , on dit que $\Lambda$ est $H$-valuée $p$ à $p$ si toute partie à $p$ éléments de $\Lambda$ est $H$-valuée.

- Un triplet de $\Lambda$ est dit $H$-antivalué si pour tout choix des $f_{a}$ et $v_{a}$ pour $a \in \Lambda$, on peut trouver un couple $(b, c)$ du triplet tel que $f_{b}\left(v_{c}\right) \notin H$.

- $\Lambda$ est dite $H$-antivaluée 3 à 3 si tous les triplets sont $H$-antivalués.

Pour le cas des triplets, on définit en outre sa valeur :

DÉfinition 3.8. - On appelle valeur d'un triplet $(a, b, c)$ de $\mathbb{Q}(V)$ le produit

$$
\epsilon_{a b c}=\zeta_{a b} \zeta_{b c} \zeta_{c a} \text {. }
$$

Cette valeur ne dépend pas des choix effectués, car $S$ est un groupe fini d'ordre $d$. On vérifie alors que le triplet $(a, b, c)$ est $H$-valué (respectivement $H$-antivalué) si et seulement si $\epsilon_{a b c} \in\{0,1\} \subset S \cup\{0\}$ (respectivement $((S \backslash$ $\{1\}) \cup\{0\}))$.

On peut donner un critère de $H$-valuation :

Lemme 3.9. - Soit $\Lambda$ une partie de $\mathbb{P}(V) \times \mathbb{P}\left(V^{*}\right)$ contenant plus de 4 éléments, telle qu'il existe $\Lambda^{\prime} \subset \Lambda$ dense et transverse. Alors $\Lambda$ est $H$-valué si et seulement si pour tout quadruplet $(a, b, c, e)$ de $\Lambda$, le produit $\eta_{a c}^{d-1} \eta_{b e}^{d-1} \eta_{a e} \eta_{b c}$ vaut 0 ou 1.

En particulier $\Lambda$ est $H$-valué si et seulement si il est $H$-valué 4 à 4 .

Démonstration. - On peut supposer que $\Lambda$ est transverse.

On voit que le produit considéré $\eta_{a c}^{d-1} \eta_{b e}^{d-1} \eta_{a e} \eta_{b c}$ ne dépend pas des choix faits. Donc si $\Lambda$ est $H$-valuée, ces produits valent 0 ou 1.

Pour la réciproque, on a par hypothèses que pour tout $(a, b, c, e)$,

$$
\eta_{a c}^{d-1} \eta_{b e}^{d-1} \eta_{a e} \eta_{b c}=1
$$

Si $\Lambda$ a quatre éléments $a, b, c$ et $e$, alors en appliquant l'hypothèse aux quadruplets $(a, b, c, e),(c, a, b, e)$ et $(b, c, a, e)$ puis en faisant le produit des égalités obtenues, on obtient :

$$
\eta_{a c}^{d-1} \eta_{c a} \eta_{b a}^{d-1} \eta_{a b} \eta_{c b}^{d-1} \eta_{b c}=1
$$

Donc si on choisit $v_{a}$ arbitrairement, on peut faire les choix de $f_{a}, v_{b}, f_{b}$, $v_{c}$ et $f_{c}$ tels que on a : $\eta_{a c}=\eta_{c a}=\eta_{b a}=\eta_{a b}=\eta_{c b}=\eta_{b c}=1$. On peut choisir de plus $v_{e}$ et $f_{e}$ tels que on a $\eta_{a e}=\eta_{e a}=1$. Les égalités données 
par l'hypothèse assurent alors que $\eta_{e c}=\eta_{c e}=\eta_{b e}=\eta_{e b}=1$. Il suffit enfin d'appliquer l'hypothèse aux quadruplets du type $(a, b, a, c)$ pour montrer que $\eta_{a a}=\eta_{b b}=\eta_{c c}=\eta_{e e}=1$. Donc $\Lambda$ est bien $H$-valuée.

Si maintenant $\Lambda$ a plus de quatre éléments, on commence par choisir un $a$ arbitrairement, et on fixe $v_{a}$. On peut alors faire des choix pour toute partie de $\Lambda$ à quatre éléments contenant $a$. On vérifie alors que tous ces choix sont cohérents en regardant des parties qui ont trois éléments en commun. On en déduit que $\Lambda$ est $H$-valué.

3.3. $H$-valuation 3 à 3. - La $H$-valuation 3 à 3 joue un rôle particulièrement important. On peut donner un critère de $H$-valuation 3 à 3 :

Lemme 3.10. - Soit $\Lambda$ une partie de $\mathbb{Q}(V)$ qui contient une partie $\Lambda^{\prime}$ dense et transverse. Alors $\Lambda$ est $H$-valuée 3 à 3 si et seulement si on peut faire le choix des vecteurs $v_{a}$ et $f_{a}$ tels que pour tout couple $(a, b)$ de points de $\Lambda$, on a $\zeta_{a b} \in\{0,1\}$.

Démonstration. - On peut à nouveau supposer $\Lambda$ transverse.

$\mathrm{Si}$ on peut faire les choix comme dans l'énoncé du lemme, alors pour tout triplet $(a, b, c)$ d'éléments de $\Lambda$, on a $\epsilon_{a b c}=1$. Donc $\Lambda$ est $H$-valuée 3 à 3 .

Réciproquement, si $\Lambda$ est $H$-valuée 3 à 3 , alors pour tout triplet $(a, b, c)$ d'éléments de $\Lambda$, on a $\epsilon_{a b c}=1$. On fixe un $a$ dans $\Lambda$ et on choisit $v_{a}$ ainsi que tous les $f_{b}$ arbitrairement. On choisit alors les vecteurs $v_{b}$ tels que si $b \neq a$, alors $\zeta_{a b}=1$. En utilisant l'hypothèse, on voit que si $b$ et $c$ sont deux éléments distincts de $\Lambda$, alors $\zeta_{b c}=1$.

\section{Action sur la $H$-sphère et $H$-proximalité}

L'étude de l'action d'un élément proximal $g$ de $\mathrm{GL}(n, k)$ sur l'espace projectif ne suffit pas pour décider si sa valeur propre $\lambda_{1}(g)$ est dans $H$.

Pour cela il faut regarder un espace plus gros qu'on appelle la $H$-sphère. On définit cette $H$-sphère, notée $\mathbb{S}(V)$ comme le quotient de $V \backslash\{0\}$ par l'action naturelle de $H$ par homothéties :

$$
\mathbb{S}(V)=(V \backslash\{0\}) / H .
$$

C'est un revêtement à $d$ feuillets de $\mathbb{P}(V)$. On peut définir une distance sur $\mathbb{S}(V)$ à valeur dans $\mathbb{R}$ de la façon suivante :

Soit $|H|$ l'ensemble des normes d'éléments de $H$. Soient $x \in u$ et $y \in v$.

On pose $D(u, v)$ égal à 3 si $\frac{\|x\|}{\|y\|}$ n'est pas dans $|H|$ (ça ne dépend pas du choix de $x$ et $y$ ) et à $\frac{1}{\|x\|} \inf \left\{\left\|x-y^{\prime}\right\|\right.$ pour $y^{\prime} \in v$, avec $\left.\left\|y^{\prime}\right\|=\|x\|\right\}$, sinon. A 
nouveau cela ne dépend pas du choix de $x$ et de $y$, et on voit que dans ce cas $D(u, v) \leq 1$.

Grâce à cette distance on peut construire des relevés de boules de l'espace projectif sur $\mathbb{S}(V)$ de la façon suivante : si $x$ appartient à $\mathbb{P}(V)$, et que $u \in \mathbb{S}(V)$ se projette sur $x$, alors la boule de centre $u$ et de rayon $\varepsilon$ de $\mathbb{S}(V)$ se projette bijectivement sur la boule de centre $x$ et de rayon $\varepsilon$ (pour $\varepsilon<1$ ).

4.1. Lien entre l'action sur la $H$-sphère et proximalité. - Définissons tout d'abord la notion de $H$-proximalité :

DÉfinition 4.1 ( $H$-proximalité). - Un élément $g$ de $\mathrm{SL}(n, k)$ est $H$-proximal (resp. $H$-biproximal, resp. $H$-loxodromique) s'il est proximal (resp. biproximal, loxodromique) et que $\lambda_{1}(g)$ est dans $H$ (resp. $\left\{\lambda_{1}(g), \lambda_{n}(g)\right\}$ est dans $H$, resp. toutes les valeurs propres sont dans $H$ ).

On dit de même qu'un sous-groupe $\Gamma$ de $\mathrm{SL}(n, k)$ est $H$-proximal (resp. $H$-loxodromique) s'il est proximal (resp. loxodromique) et que tout élément proximal (resp. loxodromique) est $H$-proximal (resp. $H$-loxodromique).

On a vu qu'un élément de $\operatorname{SL}(n, k)$ est proximal si et seulement si son action sur l'espace projectif a un point fixe attracteur. On remarque de même que cet élément est $H$-proximal si et seulement s'il a un point fixe attracteur dans $\mathbb{S}(V)$. Il en a alors exactement $d$.

On peut alors continuer cette analyse et étudier l'action des groupes $H$-proximaux sur la $H$-sphère. Soit $\Gamma$ un sous-groupe Zariski-dense de $\operatorname{SL}(n, k)$. On appelle $\Lambda_{\Gamma}^{\mathbb{S}}$ le relevé sur la $H$-sphère de l'ensemble $\Lambda_{\Gamma}^{\mathbb{P}}$

On obtient alors la propriété suivante :

Propriété 2. - Considérons $\Gamma$ un sous-groupe $\mathbb{Q}_{p}$-Zariski-dense de $\operatorname{SL}(n, k)$. Alors $\Gamma$ est $H$-proximal si et seulement si il existe un fermé $\Gamma$-invariant $F$ de $\Lambda_{\Gamma}^{\mathbb{S}}$ tel que $\Lambda_{\Gamma}^{\mathbb{S}}$ est l'union disjointe des ensembles $(\alpha F)_{\alpha \in S}$.

Dans ce cas l'action de $\Gamma$ sur $F$ est minimale.

Démonstration. - Supposons qu'on a ce fermé $F$ et cette décomposition de $\Lambda_{\Gamma}^{\mathbb{S}}$. Soit alors $g$ un élément proximal de $\Gamma$. On sait que $x_{g}^{+}$appartient à $\Lambda_{\Gamma}^{\mathbb{P}}$. Soit $\alpha$ la classe de la valeur propre $\lambda_{1}(g)$ dans $S$. Soit $x^{+} \in F$ un relevé de $x_{g}^{+}$. Alors on a par hypothèse $g\left(x^{+}\right)=\alpha x^{+} \in F$. Donc $\alpha=1$ et $g$ est $H$-proximal.

Réciproquement, supposons que $\Gamma$ est $H$-proximal. Soit $F$ un fermé minimal de $\Lambda_{\Gamma}^{\mathbb{S}}$ pour l'action de $\Gamma$. On sait que l'action de $\Gamma$ sur $\Lambda_{\Gamma}^{\mathbb{P}}$ est minimale, donc $F$ se projette sur $\Lambda_{\Gamma}^{\mathbb{P}}$. Donc on sait que $\Lambda_{\Gamma}^{\mathbb{S}}$ est l'union des $(\alpha F)_{\alpha \in S}$. Il reste à montrer que cette union est disjointe.

Soit donc $\alpha \in S$ tel que $\alpha F \cap F \neq \varnothing$. Alors par minimalité, on obtient $\alpha F=F$. Soit $g$ un élément proximal de $\Gamma$, et $x^{+} \in F$ un relevé de $x_{g}^{+}$. L'orbite sous $\Gamma$ de $x^{+}$est dense dans $F$, donc il existe $h \in \Gamma$ tel que $h\left(x^{+}\right)$est très proche 
de $\alpha x^{+}$. Alors pour $n$ grand, $h_{n}=g^{n} h g^{n}$ est proximal, donc $H$-proximal. Or $x_{h_{n}}^{+}$est très proche de $x_{g}^{+}$. Soit donc $y^{+}$proche de $x^{+}$un relevé de $x_{h_{n}}^{+}$. Alors $h_{n}\left(y^{+}\right)$est très proche de $\alpha x^{+}$. Donc, comme $H$ est ouvert, $\lambda_{1}\left(h_{n}\right)$ a pour classe $\alpha$ dans $S$. Donc $\alpha=1$, et l'union est bien disjointe.

D'autre part, on voit que dans ces conditions l'action de $\Gamma$ sur $F$ est bien minimale.

4.2. Sous-groupes $H$-proximaux. - La question de l'existence de sous-groupes $H$-proximaux est un premier pas pour traiter simultanément toutes les valeurs propres. Nous avons alors le théorème suivant, dont le théorème 1.5 est une conséquence directe :

THÉORÈme 4.2. - Soit $k$ une extension finie de $\mathbb{Q}_{p}$ et $H$ un sous-groupe d'indice fini de $k^{*}$.

Alors les trois propositions suivantes sont équivalentes :

(1) $\mathrm{SL}(n, k)$ admet un sous-groupe $\mathbb{Q}_{p}$-Zariski-dense dont tous les éléments sont $H$-proximaux.

(2) $\mathrm{SL}(n, k)$ admet un sous-groupe $\mathbb{Q}_{p}$-Zariski-dense et $H$-proximal.

(3) -1 appartient à $H$ ou $n$ est différent de 2.

Nous allons tout d'abord énoncer un autre résultat, qui nous permettra de montrer le théorème.

Proposition 4.3. - Considérons $\Gamma$ un sous-groupe proximal et $\mathbb{Q}_{p}$-Zariskidense de $\mathrm{SL}(n, k)$. Alors les cinq conditions suivantes sont équivalentes :

(1) $\Gamma$ contient un sous-groupe $\mathbb{Q}_{p}$-Zariski-dense dont tous les éléments sont $H$-proximaux.

(2) $\Gamma$ contient un sous-groupe $\mathbb{Q}_{p}$-Zariski-dense et $H$-proximal.

(3) $\Gamma$ contient un sous-groupe $\mathbb{Q}_{p}$-Zariski-dense $\Delta$ tel que $\Lambda_{\Delta}^{\mathbb{Q}(V)}$ est $H$-valué.

(4) $\Lambda_{\Gamma}^{\mathbb{Q}(V)}$ n'est pas $H$-antivalué trois à trois.

(5) $\Lambda_{\Gamma}^{\mathbb{Q}(V)}$ contient une partie infinie transverse $H$-valuée.

Montrons dans un premier temps que cette proposition implique le théorème.

Démonstration. - L'équivalence entre le premier et le deuxième point du théorème est dans la proposition. Il reste donc à prouver que $\mathrm{SL}(n, k)$ contient des sous-groupes $\mathbb{Q}_{p}$-Zariski-denses et $H$-proximaux si et seulement si $n$ est différent de 2 ou bien -1 est dans $H$.

Commençons par un lemme qui permet de comprendre pourquoi la condition est nécessaire

Lemme 4.4. - Soit $\Gamma$ un sous-groupe proximal et $\mathbb{Q}_{p}$-Zariski-dense de $\mathrm{SL}(n, k)$ préservant une forme bilinéaire $b$. Alors on a l'alternative suivante : 
- si b est symétrique, alors $\Lambda_{\Gamma}^{\mathbb{Q}(V)}$ est $H$-valué.

- sib est antisymétrique, alors si-1 n'appartient pas à $H, \Lambda_{\Gamma}^{\mathbb{Q}(V)}$ est $H$-antivalué 3 à 3.

Démonstration. - Soit $b$ la forme bilinéaire préservée. Notons alors $K=\{g \in$ $\operatorname{SL}(n, k) / \forall v, w: b(g v, g w)=b(v, w)\}$. On peut supposer $\Gamma=K$. Alors l'ensemble $\Lambda_{\Gamma}^{\mathbb{Q}(V)}$ est l'orbite fermée de $K$ dans $\mathbb{Q}(V)$. Or cette orbite est définie comme suit : pour tout $v \in V$, on note $v^{*}$ la forme linéaire $v^{\prime} \mapsto b\left(v, v^{\prime}\right)$. Alors $\Lambda_{\Gamma}^{\mathbb{Q}(V)}=\left\{\left(v, v^{*}\right) / v \in V, b(v, v)=0\right\}$ s'identifie à l'ensemble des droites isotropes.

On choisit donc pour tout $a$ dans $\Lambda_{\Gamma}^{\mathbb{Q}(V)}$ un vecteur $v_{a}$ arbitraire, et on pose $f_{a}=b\left(v_{a},.\right)$.

Alors, dans le premier cas, pour tout couple $(a, b)$, on a $\zeta_{a b}=1$. Donc pour tout triplet $(a, b, c), \epsilon_{a b c}=1$ ou 0 et $\Lambda_{\Gamma}^{\mathbb{Q}(V)}$ est $H$-valué.

Et, dans le deuxième cas, pour tout couple $(a, b)$, on a $\zeta_{a b}=-1$. Donc pour tout triplet $(a, b, c), \epsilon_{a b c}=-1$ ou 0 . Donc, si -1 n'est pas élément de $H, \Lambda_{\Gamma}^{\mathbb{Q}(V)}$ est $H$-antivalué.

Montrons maintenant le théorème. Nous distinguons le cas de la dimension 2.

CAS $1: n=2$

En dimension $2, \mathrm{SL}(2, k)$ préserve une forme bilinéaire symplectique. Donc pour $n=2$, l'existence d'un groupe $\Gamma \mathbb{Q}_{p}$-Zariski-dense et $H$-proximal implique que -1 appartienne à $H$. Réciproquement, si -1 appartient à $H$, vu les choix que l'on peut faire, pour tout $\Gamma \mathbb{Q}_{p}$-Zariski-dense dans $\operatorname{SL}(2, k), \Lambda_{\Gamma}^{\mathbb{Q}(V)}$ est $H$-valué et donc on vérifie les conditions de la proposition 4.3.

CAS $2: n \geq 3$

Il suffit maintenant de construire un triplet $H$-valué pour $\operatorname{SL}(n, k)$. En effet, si $\Lambda_{\mathrm{SL}(n, k)}^{\mathbb{Q}(V)}$ contient ce triplet, cet ensemble $\Lambda_{\mathrm{SL}(n, k)}^{\mathbb{Q}(V)}$ n'est en particulier pas $H$-antivaluée trois à trois. Alors la proposition 4.3 implique que $\operatorname{SL}(n, k)^{(4)}$ admet un sous-groupe $\mathbb{Q}_{p}$-Zariski-dense et $H$-proximal, ce qui est la conclusion souhaitée.

Soit donc $\left(e_{1}, e_{2}, \ldots, e_{n}\right)$ une base de $k^{n}$. Soit $a$ un élément de $H$ de norme plus grande strictement que 1.

(4) Ce groupe est bien par ailleurs proximal et $\mathbb{Q}_{p}$-Zariski-dense dans $\operatorname{SL}(n, k)$ ! 
Soit $g \in \mathrm{SL}(n, k)$ dont la matrice dans cette base est :

$$
\left(\begin{array}{lllll}
a & & & \\
& 1 & & 0 & \\
& & \ddots & & \\
& 0 & & 1 & \\
& & & & a^{-1}
\end{array}\right)
$$

Soit maintenant $h \in \mathrm{SL}(n, k)$ qui est représenté par la même matrice mais cette fois dans la base $\left(e_{1}+2 e_{2}+e_{n}, e_{1}-e_{n}, \ldots, e_{n}\right)$.

Alors un calcul rapide montre que le triplet $\left(a_{g}^{+}, a_{g}^{-}, a_{h}^{+}\right)$est $H$-valué. Il suffit en effet de faire les choix suivants pour les représentants de $a_{g}^{+}, a_{g}^{-}$et $a_{h}^{+}$:

$-a_{g}^{+}=\left[\left(e_{1}, e_{n}^{*}\right)\right]$

$-a_{g}^{-}=\left[\left(e_{n}, e_{1}^{*}\right)\right]$

$-a_{h}^{+}=\left[\left(e_{1}+2 e_{2}+e_{n}, e_{1}^{*}+e_{n}^{*}-e_{2}^{*}\right)\right]$

On a bien montré le théorème 4.2 grâce à la proposition 4.3.

4.3. Démonstration de la proposition 4.3. - Il faut donc maintenant démontrer la proposition 4.3. Nous aurons besoin pour cela de trois lemmes.

Voyons tout d'abord comment construire une partie infinie $H$-valuée de l'ensemble limite dans $\mathbb{Q}(V)$ à partir d'un triplet $H$-valué :

LEMME 4.5. - Soient $g$ un élément biproximal de $\mathrm{GL}\left(k^{n}\right)$ et a appartenant à $\mathbb{Q}(V)$ tels que le triplet $\left(a, a_{g}^{+}, a_{g}^{-}\right)$soit transverse et $H$-valué. Alors il existe un entier $i>1$ tel que l'ensemble $\left\{g^{i m} . a / m \in \mathbb{Z}\right\}$ est transverse et $H$-valué.

Démonstration. - On suppose - quitte à remplacer $g$ par $g^{d}$ - que $g$ et $g^{-1}$ sont $H$-proximaux.

On fait les choix des vecteurs $v, f, v_{g}^{ \pm}$et $f_{g}^{ \pm}$de telle sorte que pour tout couple $(b, c)$ du triplet $\left(a, a_{g}^{+}, a_{g}^{-}\right)$, on a $\eta_{b c}=f_{b}\left(v_{c}\right)=1$. C'est possible car le triplet est transverse et $H$-valué.

Notons $v_{m}=g^{m} v$ et $f_{m}=g^{m} f$. Travaillons dans la $H$-sphère $\mathbb{S}(V)$ et pour $x$ dans $V$ notons $\tilde{x}$ sa projection dans $\mathbb{S}(V)$. Alors, comme $f_{g}^{-}(v)=f_{g}^{-}\left(v_{g}^{+}\right)$, on sait que $\tilde{v}_{n}$ tend vers $\tilde{v}_{g}^{+}$quand $n \rightarrow+\infty$. En effet $\tilde{v}_{n}$ tend vers $\alpha \tilde{v}_{g}^{+}$pour un $\alpha \in S$, et la condition impose $\alpha=1$. De même, $\tilde{v}_{n}$ tend vers $\tilde{v}_{g}^{-}$quand $n \rightarrow-\infty$. L'ouverture de $H$ nous garantit alors l'existence de $i_{0}$ tel que pour tout entier $i$ avec $|i| \geq i_{0}, f\left(v_{i}\right) \in H$. Mais alors si $i$ et $j$ sont deux entiers tels que $|i-j| \geq i_{0}$, on a $f_{j}\left(v_{i}\right) \in H$. Donc la famille $\left\{g^{i_{0} m} . a / m \in \mathbb{Z}\right\}$ est transverse et $H$-valuée.

Il faut maintenant arriver à construire des sous-groupes Zariski-dense. C'est l'objet du second lemme, qui est très proche du lemme 7.2 de [2].

TOME $139-2011-\mathrm{N}^{\mathrm{O}} 2$ 
Lemme 4.6. - Soit $\Gamma$ un sous-groupe $\mathbb{Q}_{p}$-Zariski-dense et proximal de $\mathrm{SL}(n, k)$. Soit $a_{1}^{ \pm}, a_{2}^{ \pm}, \ldots, a_{m}^{ \pm}, \ldots$ une famille infinie d'éléments de $\Lambda_{\Gamma}^{\mathbb{Q}}$. Alors pour tout $\epsilon>0$, on peut trouver des éléments $g_{1}, \ldots, g_{m}$ de $\Gamma$ tels que:

- Pour tout $1 \leq i \leq m, g_{i}$ est $H$-biproximal.

- Pour tout $1 \leq i \leq m, d\left(a_{g_{i}}^{+}, a_{i}^{+}\right)<\epsilon$ et $d\left(a_{g_{i}}^{-}, a_{i}^{-}\right)<\epsilon$.

- Pour tout $p \geq 1$, le sous-groupe $\Delta_{p}$ de $\Gamma$ engendré par $\left(g_{i}^{p}\right)_{1 \leq i \leq m}$ est $\mathbb{Q}_{p}$-Zariski-dense dans $\Gamma$.

Démonstration. - On construit la suite $g_{i}$ par récurrence : d'après la proposition 3.6, on peut trouver $g_{1} \in \Gamma$ biproximal tel que $d\left(a_{g_{1}}^{+}, a_{1}^{+}\right)<\epsilon$ et $d\left(a_{g_{1}}^{-}, a_{1}^{-}\right)<\epsilon$. Quitte à remplacer $g_{1}$ par $g_{1}^{d}$, on peut supposer que $g_{1}$ est $H$-biproximal. On note $\Gamma_{1}$ la $\mathbb{Q}_{p}$-adhérence de Zariski du groupe engendré par $g_{1}$. Quitte à remplacer $g_{1}$ par une puissance, on peut supposer que $\Gamma_{1}$ est $\mathbb{Q}_{p}$-Zariski-connexe.

Supposons construits $\left(g_{1}, \ldots, g_{j-1}\right)$ appartenant à $\Gamma, H$-biproximaux, vérifiant que la $\mathbb{Q}_{p}$-adhérence de Zariski du groupe que chacun engendre est $\mathbb{Q}_{p}$-Zariski-connexe, et tels que : pour tout $1 \leq i \leq j-1, d\left(a_{g_{i}}^{+}, a_{i}^{+}\right)<\epsilon$ et $d\left(a_{g_{i}}^{-}, a_{i}^{-}\right)<\epsilon$. On note $\Gamma_{j-1}$ la $\mathbb{Q}_{p}$-adhérence de Zariski dans $\Gamma$ du groupe engendré par ces éléments. C'est un $\mathbb{Q}_{p}$-fermé $\mathbb{Q}_{p}$-Zariski-connexe. Si $\Gamma_{j-1}=\Gamma$, alors la famille cherchée est construite. On suppose donc que $\Gamma_{j-1}$ n'est pas égal à $\Gamma$.

D'après la proposition 3.6, l'ensemble des éléments biproximaux tels que $d\left(a_{g}^{+}, a_{j}^{+}\right)<\epsilon$ et $d\left(a_{g}^{-}, a_{j}^{-}\right)<\epsilon$ est $\mathbb{Q}_{p}$-Zariski-dense dans $\Gamma$. Donc on peut choisir dans cet ensemble un élément $g_{j} H$-biproximal, en dehors de $\Gamma_{j-1}$ et engendrant un groupe $\mathbb{Q}_{p}$-Zariski-connexe.

La suite $\left(\Gamma_{j}\right)$ est une suite croissante d'ensembles $\mathbb{Q}_{p}$-Zariski-fermés et $\mathbb{Q}_{p}$-Zariski-connexes. Elle stationne donc et il existe $m$ tel que $\Gamma_{m}=\Gamma$. De plus les propriétés de $\mathbb{Q}_{p}$-Zariski-connexité nous assurent que pour tout $p \geq 1$, la $\mathbb{Q}_{p}$-adhérence de Zariski de $\Delta_{p}$ contient $\Gamma_{m}=\Gamma$.

Un dernier lemme permettra de vérifier que les groupes construits dans le lemme précédent sont composés d'éléments $H$-proximaux.

Lemme 4.7. - Soit $E$ une partie finie de $\mathrm{GL}(V)$ composée d'éléments $H$-biproximaux et telle que l'inverse de tout élément de $E$ est encore dans E. Supposons que l'ensemble $\Lambda_{E}^{\mathbb{Q}}=\left\{a_{g}^{+} / g \in E\right\}$ de $\mathbb{Q}(V)$ est transverse. De plus, on suppose que pour tout $i \geq 1$ le sous groupe $\Delta_{i}$ de $\mathrm{GL}(V)$ engendré par $\left\{g^{i} / g \in E\right\}$ agit irréductiblement sur $V$. Alors on peut trouver un entier $i_{0} \geq 1$ tel que, pour tout $i \geq i_{0}$, on $a$ :

- Tous les éléments de $\Delta_{i}-\{e\}$ sont proximaux.

- $\Delta_{i}$ est $H$-proximal si et seulement si $\Lambda_{E}^{\mathbb{Q}}$ est $H$-valué.

$-\Lambda_{\Delta_{i}}^{\mathbb{Q}}$ est $H$-valué si et seulement si $\Lambda_{E}^{\mathbb{Q}}$ est $H$-valué. 
Démonstration. - Soit $\epsilon$ un réel positif. Soit $g$ un élément proximal, on dispose alors de $x_{g}^{+}$et $y_{g}^{-}$, et on note $Y_{g}^{-}$le noyau de $y_{g}^{-}$. On définit alors $b_{\epsilon}(g)=\{x \in$ $\left.\mathbb{P}(V) / d\left(x, x_{g}^{+}\right)<\epsilon\right\}$ et $B_{\epsilon}(g)=\left\{x \in \mathbb{P}(V) / d\left(x, Y_{g}^{-}\right)>\epsilon\right\}$.

On dit alors que $g$ est $\epsilon$-proximal si on a : $b_{\epsilon}(g) \subset B_{\epsilon}(g), g\left(B_{\epsilon}(g)\right) \subset b_{\epsilon}(g)$ et l'action de $g$ restreinte à $B_{\epsilon}(g)$ est $\epsilon$-lipschitzienne.

Fixons $\epsilon$ suffisamment petit tel que, pour tout $g$ et $h$ distincts de $E$ avec $g \neq h^{-1}$, on a $b_{\epsilon}(h) \subset B_{\epsilon}(g)$. On peut trouver un entier $i_{0}$ tel que tous les $g^{i_{0}}$ pour $g$ un élément de $E$ sont $\epsilon$-proximaux. On note $E^{i}=\left\{g^{i} / g \in E\right\}$ pour tout entier $i$.

Soit $i \geq i_{0}$. Soit $h=h_{l} \cdots h_{1}$ un élément de $\Delta_{i}$, avec $h_{k}$ dans $E^{i}$. On suppose (quitte à simplifier et à conjuguer) que pour tout $1 \leq k \leq l, h_{k} \neq h_{k+1}^{-1}$, avec la notation $h_{1}=h_{l+1}$. Comme les $h_{k}$ sont dans $E^{i}$, ils sont $\epsilon$-proximaux.

Dans ces conditions, on vérifie que pour tout $1 \leq k \leq l$, on a les inclusions suivantes : $h_{k} \cdots h_{1}\left(b_{\epsilon}\left(h_{l}\right)\right) \subset h_{k} \cdots h_{1}\left(B_{\epsilon}\left(h_{1}\right)\right) \subset b_{\epsilon}\left(h_{k}\right)$. Donc on obtient que $h\left(b_{\epsilon}\left(h_{l}\right)\right) \subset b_{\epsilon}\left(h_{l}\right)$ et on voit que la restriction de $h$ à $b_{\epsilon}\left(h_{l}\right)$ est $\epsilon$-lipschitzienne. Donc $h$ admet un point fixe attracteur dans $b_{\epsilon}\left(h_{l}\right)$ et est donc proximal.

Le premier point est donc démontré.

Pour le deuxième point, il va falloir à nouveau travailler dans la $H$-sphère. Pour tout $g$ dans $E$, choisissons $v_{g}^{+}$et $f_{g}^{-}$des relevés de $x_{g}^{+}$et $y_{g}^{-}$dans respectivement $V$ et $V^{*}$ tels que $f_{g}^{-}\left(v_{g}^{+}\right) \in H$. Si $x$ appartient à $V$, on note $\tilde{x}$ son projeté dans la $H$-sphère. Soit $a_{\epsilon}(g)=\left\{v \in \mathbb{S}(V) / D\left(v, \tilde{v}_{g}^{+}\right)<\epsilon\right\}$. Il est clair par construction que $a_{\epsilon}(g)$ se projette sur $b_{\epsilon}(g)$. Donc pour tout $h$ et $h^{\prime}$ distincts de $E^{i}$ avec $h^{\prime} \neq h^{-1}$, on peut définir $\eta_{h h^{\prime}}$ comme l'unique élément de $S$ tel que on a $h\left(a_{\epsilon}\left(h^{\prime}\right)\right) \subset \eta_{h h^{\prime}} a_{\epsilon}(h)$. On voit que $\eta_{h h^{\prime}}$ est la classe de $f_{h}^{-}\left(v_{h^{\prime}}^{+}\right)$.

Si maintenant, comme pour le point précédent, on a un mot $h=h_{l} \cdots h_{1}$ réduit avec $h_{k}$ dans $E^{i}$, on définit $\eta_{h}=\eta_{h_{1} h_{l}} \cdots \eta_{h_{2} h_{1}}$. On a alors $h\left(a_{\epsilon}\left(h_{l}\right)\right) \subset$ $\eta_{h} a_{\epsilon}\left(h_{l}\right)$.

Donc $\Delta_{i}$ est $H$-proximal si et seulement si on a $\forall h \in \Delta_{i} \backslash\{e\}, \eta_{h}=1$. D'après le lemme 3.9, on voit que c'est équivalent au fait que $\Lambda_{E}^{\mathbb{Q}}$ est $H$-valué.

Pour le troisième point, on remarque tout d'abord que $\Lambda_{E}^{\mathbb{Q}}$ est inclus dans $\Lambda_{\Delta_{i}}^{\mathbb{Q}}$. Il suffit donc de montrer que si $\Lambda_{E}^{\mathbb{Q}}$ est $H$-valué, alors $\Lambda_{\Delta_{i}}^{\mathbb{Q}}$ l'est aussi. Raisonnons par l'absurde. Si $\Lambda_{E}^{\mathbb{Q}}$ est $H$-valué, $\Delta_{i}$ est $H$-proximal. Mais si $\Lambda_{\Delta_{p}}^{\mathbb{Q}}$ n'est pas $H$-valué, on peut construire grâce aux deux premiers points de ce lemme et au lemme précédent un élément proximal non $H$-proximal de $\Delta_{i} \backslash\{e\}$. C'est la contradiction cherchée.

Nous pouvons maintenant démontrer l'équivalence de la proposition 4.3 :

Démonstration. - Le premier point implique clairement le second.

Avec le lemme 4.7 et sa démonstration, on voit que le deuxième point implique le troisième. 
Du troisième point on en déduit facilement le quatrième : on dispose d'une partie infinie $H$-valuée, donc a fortiori un triplet.

Pour passer du quatrième point au cinquième, on utilise le lemme 4.5 : soit $\left(a, a^{+}, a^{-}\right)$un triplet transverse $H$-valué. D'après le lemme 3.6 on trouve un élément biproximal $g$ tel que $a_{g}^{+}$est très proche de $a^{+}$et $a_{g}^{-}$de $a^{-}$. Donc on dispose du triplet $\left(a, a_{g}^{+}, a_{g}^{-}\right)$transverse et $H$-valué. Ce qui permet d'appliquer le lemme 4.5 pour avoir une partie infinie transverse $H$-valuée.

Enfin le cinquième implique le premier point : Soit une famille $a_{1}^{ \pm}, \ldots, a_{m}^{ \pm}$ transverse $H$-valuée. On choisit un $\epsilon>0$ suffisamment petit et $g_{1}, \ldots, g_{m}$ comme dans le lemme 4.6 de sorte que la famille $a_{g_{1}}^{ \pm}, \ldots, a_{g_{m}}^{ \pm}$est encore transverse et $H$-valuée. Soit $\Delta_{i}$ le groupe engendré par les $g_{j}^{i}$. D'après les lemmes 4.6 et 4.7, pour $i$ suffisamment grand, $\Delta_{i}$ est $\mathbb{Q}_{p}$-Zariski-dense composé d'éléments $H$-proximaux.

Nous avons donc compris le comportement de la première valeur propre. Avec le même genre d'idée, on peut traiter toutes les valeurs propres simultanément. Ainsi dans la prochaine partie, nous étudierons les conditions d'existence de sous-groupes $H$-loxodromiques de $\operatorname{SL}(n, k)$.

\section{Sous-groupes $H$-loxodromiques}

5.1. La variété des drapeaux. - Le but de cette partie est de démontrer le théorème suivant, qui est un peu plus précis que le theorème 1.6 de l'introduction.

THÉORÈme 5.1. - Soit $k$ une extension finie de $\mathbb{Q}_{p}$ et $H$ un sous-groupe d'indice fini de $k^{*}$.

Alors les trois propositions suivantes sont équivalentes :

(1) $\mathrm{SL}(n, k)$ contient un sous-groupe $\mathbb{Q}_{p}$-Zariski-dense dont tous les éléments sont $H$-loxodromiques.

(2) $\mathrm{SL}(n, k)$ contient un sous-groupe $H$-loxodromique et Zariski-dense.

(3) -1 appartient à $H$, ou $n$ n'est pas congru à 2 modulo 4.

La démonstration sera parallèle à la démonstration du théorème 4.2. Il faudra cependant être un peu plus précis car nous travaillons maintenant avec toutes les valeurs propres simultanément. Il faudra pour cela utiliser un soupçon de théorie des représentations.

Pour le groupe $\mathrm{SL}(n, k)$ on connait des représentations $r_{l}$ dans $V_{l}=\Lambda^{l} k^{n}$ pour $1 \leq k \leq n-1$. On a vu que si $\Gamma$ est $H$-loxodromique, alors tous les $r_{l}(\Gamma)$ sont $H$-proximaux. On fixe une base $\left(e_{1}, \ldots, e_{n}\right)$ de $V$.

Nous travaillons dans la variété des drapeaux $X \simeq \mathrm{SL}(n, k) / T^{+}$de $V$ où $T^{+}$ est l'ensemble des matrices triangulaires supérieures. Il nous faut donc définir dans ce cadre les notions que l'on avait définies pour les éléments proximaux. 
Pour cela, définissons des fonctions $\varphi_{l}$ de $X$ dans $\mathbb{Q}\left(V_{l}\right)$ pour tout $1 \leq l \leq$ $n-1$ : soit $z$ un drapeau de $X$, et $\left(f_{1}, \ldots, f_{n}\right)$ une base adaptée à $z$. On note $\left(f_{1}^{*}, \ldots, f_{n}^{*}\right)$ la base duale.

DÉfinition 5.2. - Avec les notations ci-dessus, $\varphi_{l}(z)$ est défini comme le point $\left(f_{1} \wedge \cdots \wedge f_{l}, f_{n}^{*} \wedge \cdots \wedge f_{n-l}^{*}\right)$ de $\mathbb{P}\left(V_{l}\right) \times \mathbb{P}\left(V_{l}^{*}\right)$.

De plus, pour tout $1 \leq l \leq n-1$, on note $a_{l}$ l'image par $\varphi_{l}$ du drapeau canonique.

La notion de transversalité dans la variété drapeau découle de ces définitions :

DÉFInItion 5.3. - Une partie $\Lambda \subset X$ est transverse si pour tout $1 \leq l \leq n-1$, $\varphi_{l}(\Lambda)$ est une partie transverse de $\mathbb{P}\left(V_{l}\right) \times \mathbb{P}\left(V_{l}^{*}\right)$.

Remarquons que si un élément $g$ de $\mathrm{SL}(n, k)$ est loxodromique, alors son action sur la variété des drapeaux $X$ a un point fixe attracteur, à savoir le drapeau engendré par la base $\left(x_{1}, \ldots, x_{n}\right)$ où $x_{i}$ est un vecteur propre associé à la valeur propre $\lambda_{i}$. Ce qui mène à la définition suivante :

DÉfinition 5.4. - Si $g$ est un élément loxodromique, on note $z_{g}^{+}$ce drapeau attracteur dans la variété $X$.

On note $z_{g}^{-}$le drapeau $z_{g^{-1}}^{+}$.

Nous pouvons maintenant énoncer une proposition similaire à la proposition 3.6 pour les éléments loxodromiques. Cette proposition complète la preuve du théorème 1.4 de l'introduction en permettant d'obtenir l'abondance des éléments loxodromiques :

Proposition 5.5. - Soit $k$ une extension finie de $\mathbb{Q}_{p}$ et $\Gamma$ un sous-groupe $\mathbb{Q}_{p}$-Zariski-dense et loxodromique de $\operatorname{SL}(n, k)$. Alors :

(1) L'ensemble $\Gamma_{\text {lox }}$ des éléments loxodromiques de $\Gamma$ est $\mathbb{Q}_{p}$-Zariski-dense dans $\Gamma$.

(2) Soit $\Lambda_{\Gamma}^{X}$ l'adhérence de l'ensemble $\left\{z_{g}^{+} / g \in \Gamma_{\text {lox }}\right\}$. Tout fermé non vide $\Gamma$-invariant de $X$ contient $\Lambda_{\Gamma}^{X}$. Donc l'action de $\Gamma$ sur $\Lambda_{\Gamma}^{X}$ est minimale.

(3) Pour tous $\varepsilon>0, z^{+}$et $z^{-}$dans $\Lambda_{\Gamma}^{X}$, l'ensemble

$\left\{g \in \Gamma_{\text {lox }} / d\left(z_{g}^{+}, z^{+}\right)<\varepsilon, d\left(z_{g}^{-}, z^{-}\right)<\varepsilon\right\}$ est encore $\mathbb{Q}_{p}$-Zariski-dense dans $\Gamma$.

(4) $\Lambda_{\Gamma}^{X}$ contient une partie dense et transverse.

Démonstration. - Comme la proposition 3.6, cette proposition est une conséquence de la partie 3 de [3]. De plus la preuve est à nouveau très similaire à celle donnée dans le cas proximal. 
5.2. $H$-valuation de l'ensemble limite. - On peut aussi définir la notion de $H$-valuation dans la variété des drapeaux :

DÉfinition 5.6. - Une partie $\Lambda$ de $X$ est $H$-valuée (resp $H$-valuée 3 à 3 ) si et seulement si, pour tout $1 \leq l \leq n-1$, son image $\varphi_{l}(\Lambda)$ dans $\mathbb{Q}\left(V_{l}\right)$ est $H$-valuée (resp $H$-valuée 3 à 3 ).

Une telle partie est $H$-antivaluée 3 à 3 si elle ne contient aucun triplet transverse $H$-valué.

On peut alors énoncer une proposition similaire à la proposition 4.3 :

Proposition 5.7. - Soit $\Gamma$ un sous-groupe $\mathbb{Q}_{p}$-Zariski-dense et loxodromique de $\mathrm{SL}(n, k)$. Alors on a l'équivalence suivante :

(1) $\Gamma$ contient un sous-groupe $\mathbb{Q}_{p}$-Zariski-dense dont tous les éléments sont $H$-loxodromiques.

(2) $\Gamma$ contient un sous-groupe $\mathbb{Q}_{p}$-Zariski-dense et $H$-loxodromique.

(3) l'ensemble limite $\Lambda_{\Gamma}^{X}$ contient un triplet transverse $H$-valué.

La démonstration de la proposition 5.7 est très similaire à celle de la proposition 4.3. Nous énonçons donc trois lemmes analogues aux trois lemmes utilisés plus haut.

LEMME 5.8. - Soient $g$ un élément loxodromique de $\mathrm{SL}(n, k)$ et $z$ appartenant $\grave{a} X$ tels que le triplet $\left(z, z_{g}^{+}, z_{g}^{-}\right)$soit $H$-valué. Alors il existe un entier $p>1$ tel que l'ensemble $\left\{g^{p n} . z / n \in \mathbb{Z}\right\}$ est transverse et $H$-valué.

Démonstration. - C'est une conséquence du lemme 4.5.

LEMME 5.9. - Soit $\Gamma$ un sous-groupe $\mathbb{Q}_{p}$-Zariski-dense loxodromique de $\mathrm{SL}(n, k)$. Soient $m \geq 2$ et $z_{1}^{ \pm}, z_{2}^{ \pm}, \ldots, z_{m}^{ \pm}, \ldots$ une famille infinie d'éléments de $\Lambda_{\Gamma}^{X}$. Alors pour tout $\epsilon>0$, on peut trouver des éléments $g_{1}, \ldots, g_{m}$ de $\Gamma$ tels que :

- Pour tout $1 \leq j \leq m, g_{j}$ est $H$-loxodromique.

- Pour tout $1 \leq j \leq m, d\left(z_{g_{j}}^{+}, z_{j}^{+}\right)<\epsilon$ et $d\left(z_{g_{j}}^{-}, z_{j}^{-}\right)<\epsilon$.

- Pour tout $i \geq 1$, le sous-groupe $\Delta_{i}$ de $\Gamma$ engendré par $\left(g_{j}^{i}\right)_{1 \leq j \leq m}$ est $\mathbb{Q}_{p}$-Zariski-dense dans $\Gamma$.

Démonstration. - La démonstration est la même que celle du lemme 4.6 (voir aussi le lemme 7.2 de [2]).

Le troisième lemme se réénonce ainsi : 
Lemme 5.10. - Soit $E$ une partie finie de $\mathrm{SL}(V)$ composée d'éléments $H$-loxodromiques et telle que l'inverse de tout élément de $E$ est encore dans $E$. Supposons que l'ensemble $\Lambda_{E}^{X}=\left\{z_{g}^{+} / g \in E\right\}$ de $X$ est transverse. De plus, on suppose que pour tout $i \geq 1$ le sous groupe $\Delta_{i}$ de $\mathrm{SL}(V)$ engendré par $\left\{g^{i} / g \in E\right\}$ est $\mathbb{Q}_{p}$-Zariski-dense dans $\mathrm{SL}(V)$. Alors on peut trouver un entier $i_{0} \geq 1$ tel que, pour tout $i \geq i_{0}$, on $a$ :

- Tous les éléments $\Delta_{i}-\{e\}$ sont loxodromiques.

- $\Delta_{i}$ est $H$-loxodromique si et seulement si $\Lambda_{E}^{X}$ est $H$-valué.

Démonstration. - C'est une conséquence du lemme 4.7.

On montre alors la proposition 5.7 de la même manière que la proposition 4.3, en remplaçant les lemmes 4.5, 4.6 et 4.7 par les trois lemmes ci-dessus.

Il reste donc à démontrer le théorème 5.1. Pour cela, le point un peu délicat est de construire un triplet $H$-valué. Nous détaillons cette construction dans la partie suivante.

5.3. Construction d'un triplet $H$-valué. - Le but de cette partie est de construire un triplet $H$-valué de la variété des drapeaux. Quand on ne s'intéressait qu'à la première valeur propre, cette construction n'était qu'une formalité. Maintenant, il faut contrôler la $H$-valuation du triplet dans toutes les représentations simultanément. Pour cela, nous utilisons l'action d'un certain élément $Y$ de $M(n, k)$.

Commençons par une définition et un lemme. On rappelle qu'on dispose de $\left(e_{1}, \ldots, e_{n}\right)$ une base de $V$.

DÉFInition 5.11. - Soient $Y$ appartenant à $\mathrm{GL}(n, k)$ et $1 \leq l \leq n-1$. Alors on note $N_{l}(Y)=\inf \left\{k \in \mathbb{N} / f_{l}\left(Y^{k} . v_{l}\right) \neq 0\right\}$, où $v_{l}=e_{1} \wedge \cdots \wedge e_{l}$ appartient à $V_{l}$ et $f_{l}=\left(e_{n-l+1} \wedge \cdots \wedge e_{n}\right)^{*}$ appartient au dual $V_{l}^{*}$ de $V_{l}$.

On remarque que $v_{l}$ et $f_{l}$ sont des vecteurs de plus haut poids restreint de $V_{l}$ et $V_{l}^{*}$ respectivement.

Le lemme suivant explique comment utiliser l'action d'un élément $Y$ nilpotent bien choisi. Comme $Y$ est supposé nilpotent, on peut passer à l'exponentielle.

LEMme 5.12. - Soient $Y$ un élément nilpotent dans $M(n, k)$ et $1 \leq l \leq n-1$. Alors on peut trouver $t$ et $t^{\prime}$ dans $H$ tels que pour tout $l$ :

(1) Si $N_{l}(Y)<\infty$, le triplet $\left(a_{l}, e^{t Y} . a_{l}, e^{t^{\prime} Y} . a_{l}\right)$ est transverse.

(2) Si $N_{l}(Y)$ est pair, il est $H$-valué.

(3) Si $N_{l}(Y)$ est impair, le triplet est $H$-valué si et seulement si -1 est dans $H$.

TOME $139-2011-\mathrm{N}^{\circ} 2$ 
Démonstration. - Soit $t_{i}$ une suite de points de $H$ tendant vers 0 . Soit maintenant $t_{i}^{\prime}$ une suite de points de $H$ tendant vers 0 tels que pour tout $i, t_{i}^{\prime}-t_{i}$ est élément de $H$ (en particulier $t_{i} \neq t_{i}^{\prime}$ ). C'est possible car $H$ est ouvert.

Fixons un entier $1 \leq l \leq n-1$.

Soit $N=N_{l}(Y)$. Soit $\left(v_{l}, f_{l}\right)$ un représentant de $a_{l}$. Calculons pour tout $i$ :

$$
\begin{aligned}
c_{t_{i} t_{i}^{\prime}} & =\left(e^{t_{i} Y} f_{l}\right)\left(e^{t_{i}^{\prime} Y} v_{l}\right) \\
& =f_{l}\left(e^{\left(t_{i}^{\prime}-t_{i}\right) Y} v_{l}\right) \\
& =\frac{\left(t_{i}^{\prime}-t_{i}\right)^{N}}{N !} f_{l}\left(Y^{N} v_{l}\right)+o\left(\left(t_{i}^{\prime}-t_{i}\right)^{N}\right)
\end{aligned}
$$

Cette expression ne s'annule pas pour $i$ grand car on a supposé que $t_{i} \neq t_{i}^{\prime}$. Donc tous les triplets $\left(a_{l}, e^{t_{i} Y} . a_{l}, e^{t_{i}^{\prime} Y} . a_{l}\right)$ sont transverses.

On peut supposer $v_{l}$ et $f_{l}$ choisis de telle sorte que $\frac{f_{l}\left(Y^{N} v_{l}\right)}{N !} \in H$. On sait alors que pour $i$ suffisamment grand $c_{t_{i} t_{i}^{\prime}}, c_{0 t_{i}}$ et $c_{0 t_{i}^{\prime}}$ sont dans $H$ car $H$ est ouvert. Il suffit donc de vérifier que $c_{t_{i} 0}, c_{t_{i}^{\prime} 0}$ et $c_{t_{i}^{\prime} t_{i}}$ sont dans $H$. C'est bien le cas si $N$ est pair, ou alors, si $N$ est impair, il faut et il suffit que -1 soit dans $H$.

Donc pour un certain $I$, on sait que le triplet $\left(a_{l}, e^{t_{I} Y} . a_{l}, e^{t_{I}^{\prime} Y} . a_{l}\right)$ vérifie les conditions du lemme.

Dans le cas où $n$ n'est pas congru à 2 modulo 4 , nous construisons maintenant un élément $Y$ de $M(n, k)$ tel que $N_{l}(Y)$ soit pair pour tout $1 \leq l \leq n-1$.

CAS $n_{1}: n$ est impair.

Posons $Y_{0} \in M(n, k)$ défini par : $Y_{0} e_{i}=e_{i+1}$ pour tout $1 \leq i \leq n$, avec $e_{n+1}=0$. On peut alors calculer $N_{l}\left(Y_{0}\right)$ pour tout $l$ : on a $N_{l}\left(Y_{0}\right)=l(n-l)$. Comme $n$ est impair, cet entier est pair pour tout $1 \leq l \leq n-1$.

CAS $n_{2}: n=2 q$ avec $q$ pair.

On définit cette fois $Y_{1}$ par $Y_{1} e_{i}=e_{i+1}+\delta_{i, q-1} e_{q+1}$ pour tout $1 \leq i \leq n$. On peut calculer, pour $1 \leq l<q, N_{l}\left(Y_{1}\right)=N_{n-l}\left(Y_{1}\right)=l(n-l-1)$. Ces entiers sont pairs.

Il reste le problème de la parité de $N_{q}\left(Y_{1}\right)$. Montrons dans un premier temps que cet entier est fini : il suffit de constater que $f_{q}\left(Y_{1}^{q^{2}} . e_{1} \wedge \cdots \wedge e_{q}\right) \neq 0$. Donc $N_{q}\left(Y_{1}\right)$ est bien fini.

On remarque alors que la représentation $r_{q}$ est orthogonale. C'est à dire que, d'après le lemme 4.4, la famille $\left(a_{q}, e^{t_{I} Y} . a_{q}, e^{t_{I}^{\prime} Y} . a_{q}\right)$ est $H$-valuée, et ce pour tout $H$ sous-groupe d'indice fini de $k^{*}$. Or il y a des tels $H$ qui ne contiennent pas -1 . Donc, d'après le lemme $5.12, N_{q}\left(Y_{1}\right)$ ne peut pas être impair. Comme il est fini, c'est donc qu'il est pair.

Tous les $N_{l}\left(Y_{1}\right)$ sont donc pairs pour $1 \leq l \leq n-1$.

Grâce à ces éléments $Y_{0}$ et $Y_{1}$, nous pouvons maintenant démontrer le théorème 5.1 . 
Preuve du théorème 5.1. - L'équivalence entre le premier point et le deuxième est déjà prouvée dans la proposition. Il reste à montrer que $\operatorname{SL}(n, k)$ contient un sous-groupe Zariski-dense et $H$-loxodromique si et seulement si -1 est dans $H$ ou $n$ n'est pas congru à 2 modulo 4

Or on remarque que la condition $n$ non congru à 2 modulo 4 est équivalente au fait qu'aucun des $r_{l}$ ne préserve une forme symplectique.

Ainsi, d'après le lemme 4.4, on voit que si -1 n'appartient pas à $H$ et $n=2 a$ est congru à 2 modulo 4 , alors $\operatorname{SL}(n, k)$ ne contient pas de sousgroupes Zariski-denses $H$-loxodromiques. En effet, la représentation $r_{a}$ est symplectique, donc $r_{a}(\mathrm{SL}(n, k))$ ne contient pas de sous-groupe $\mathbb{Q}_{p}$-Zariski-dense $H$-proximal, donc $\mathrm{SL}(n, k)$ ne contient pas de sous-groupe $\mathbb{Q}_{p}$-Zariski-dense $H$-loxodromique. Donc le premier point implique le deuxième.

Il suffit maintenant de montrer que dans les autres cas, on peut trouver des sous-groupes $\mathbb{Q}_{p}$-Zariski-denses dont tous les éléments sont $H$-loxodromiques. Il faut donc construire un triplet transverse et $H$-valué de la variété des drapeaux.

Pour cela, on sait que le drapeau canonique $z_{0}$ associé à la base $\left(e_{1}, \ldots, e_{n}\right)$ est dans $\Lambda_{\mathrm{SL}(n, k)}^{X}$. De plus, dans tous les cas considérés, le lemme 5.12 nous donne $t$ et $t^{\prime}$ dans $H$ tels que le triplet $\left(z_{0}, e^{t Y} z_{0}, e^{t^{\prime} Y} z_{0}\right)$ est transverse et $H$-valué car son image dans toutes les représentations $r_{l}$ l'est (ici $Y$ désigne $Y_{0}$ si $n$ est impair et $Y_{1}$ si $n$ est pair).

On en déduit l'existence d'un sous-groupe de $\mathrm{SL}(n, k)$ Zariski-dense dont tous les éléments sont $H$-loxodromiques d'après la proposition 5.7. Ainsi le deuxième point implique le premier.

Le théorème 5.1 est donc bien démontré.

Pour en déduire le théorème 1.1, il suffit de remarquer que grâce au théorème 2.5 et à l'équivalence entre les deux premiers points du théorème 5.1 , on a l'équivalence entre l'existence d'un sous-groupe $\mathbb{Q}_{p}$-Zariski-dense et $H$-loxodromique, et l'existence d'un sous-groupe $\mathbb{Q}_{p}$-Zariski-dense dont toutes les matrices ont leur spectre dans $H$.

Concluons en donnant une dernière application du théorème 1.1 :

Corollaire 5.13. - Pour tout entier $n$, pour toute extension finie $k$ de $\mathbb{Q}_{p}$, on peut trouver un sous-groupe $\mathbb{Q}_{p}$-Zariski-dense de $\mathrm{SL}(n, k)$ dont toutes les valeurs propres sont des cubes. 


\section{BIBLIOGRAPHIE}

[1] H. Abels, G. A. Margulis \& G. A. SoĬFer - « Semigroups containing proximal linear maps », Israel J. Math. 91 (1995), p. 1-30.

[2] Y. Benoist - «Actions propres sur les espaces homogènes réductifs », Ann. of Math. 144 (1996), p. 315-347.

[3] , «Propriétés asymptotiques des groupes linéaires », Geom. Funct. Anal. 7 (1997), p. 1-47.

[4] « « Automorphismes des cônes convexes », Invent. Math. 141 (2000), p. 149-193.

[5] _ « Convexes divisibles. II », Duke Math. J. 120 (2003), p. 97-120.

[6] _ _ Convexes divisibles. I», in Algebraic groups and arithmetic, Tata Inst. Fund. Res., 2004, p. 339-374.

[7] « « Convexes divisibles. III », Ann. Sci. École Norm. Sup. 38 (2005), p. $793-832$.

[8] — Invent. Math. 164 (2006), p. 249-278.

[9] Y. Benoist \& F. LABOURIE - « Sur les difféomorphismes d'anosov affines à feuilletages stable et instable différentiables », Inventiones mathematicae 111 (1993), p. p.471-474.

[10] N. Bourbaki - Éléments de mathématique. XXVI. Groupes et algèbres de Lie. Chapitre 1 : Algèbres de Lie, Actualités Sci. Ind. No. 1285. Hermann, Paris, 1960.

[11] I. Y. GOL'DShEÍd \& G. A. MARgulis - « Lyapunov exponents of a product of random matrices », Uspekhi Mat. Nauk 44 (1989), p. 13-60.

[12] S. LANG - Algebra, Addison-Wesley Publishing Co., Inc., Reading, Mass., 1965.

[13] V. P. Platonov - « Proximal elements in p-adic groups ».

[14] G. PRASAD - « R-regular elements in Zariski-dense subgroups », Quart. J. Math. Oxford Ser. 45 (1994), p. 541-545.

[15] J. Tits - «Free subgroups in linear groups », J. Algebra 20 (1972), p. 250270 . 